\title{
Charge interactions, reaction kinetics and dimensionality effects on electrokinetic remediation: A model-based analysis
}

Sprocati, Riccardo; Rolle, Massimo

Published in:

Journal of Contaminant Hydrology

Link to article, DOI:

10.1016/j.jconhyd.2019.103567

Publication date:

2020

Document Version

Peer reviewed version

Link back to DTU Orbit

Citation (APA):

Sprocati, R., \& Rolle, M. (2020). Charge interactions, reaction kinetics and dimensionality effects on electrokinetic remediation: A model-based analysis. Journal of Contaminant Hydrology, 229, [103567]. https://doi.org/10.1016/j.jconhyd.2019.103567

\section{General rights}

Copyright and moral rights for the publications made accessible in the public portal are retained by the authors and/or other copyright owners and it is a condition of accessing publications that users recognise and abide by the legal requirements associated with these rights.

- Users may download and print one copy of any publication from the public portal for the purpose of private study or research.

- You may not further distribute the material or use it for any profit-making activity or commercial gain

- You may freely distribute the URL identifying the publication in the public portal 
This is a Post Print of the article published online $9^{\text {th }}$ November 2019 in Journal of Contaminant Hydrology. The publishers' version is available at the permanent link:

https://doi.org/10.1016/j.jconhyd.2019.103567

\title{
Charge interactions, reaction kinetics and dimensionality effects on electrokinetic remediation: A model-based analysis
}

\author{
Riccardo Sprocati ${ }^{1}$, Massimo Rolle ${ }^{1 *}$ \\ ${ }^{1}$ Department of Environmental Engineering, Technical University of Denmark, Miljøvej, \\ Building 115, 2800 Kgs. Lyngby, Denmark
}

*Corresponding author phone: +45 45251566; email: masro@env.dtu.dk

\section{HIGHLIGHTS}

- Electrokinetic transport strongly depends on charge coupling and ion concentrations

- Impact of diffusive properties and electrolyte mixtures on electromigration

- Effects of reaction kinetics on EK-based contaminant degradation

- 2D analysis of electrode configuration for electrokinetic delivery and mass removal 


\section{ABSTRACT}

The potential of electrokinetic remediation technologies (EKR) for the removal of different contaminants from subsurface porous media has been increasingly recognized. Despite electrokinetic applications have shown promising results, quantitative understanding of such systems is still challenging, due to the complex interplay between physical transport processes, electrostatic interactions, and geochemical reactions.

In this study, we perform a model-based analysis of electrokinetic transport in saturated porous media. We investigate the effects of: (i) Coulombic interactions between ions in the system mobilized by electromigration, (ii) reaction kinetics on the overall removal efficiency of a noncharged organic contaminant, and (iii) dimensionality and different electrode configurations. The results show that such effects play a major role on the performance of electrokinetic systems. The simulations illuminate the importance of microscopic processes, such as electrostatic interactions and ion-specific diffusivities, and their non-intuitive macroscopic impact on the delivery of charged amendments and on the efficiency of contaminant removal. The insights of this study are valuable to improve and optimize the design and the operational strategies of electrokinetic remediation systems. 


\section{Introduction}

In-situ remediation of contaminated sites is a difficult challenge, since the displacement of contaminants and the delivery of amendments is often hampered by mass transfer limitations in low-permeability zones where transport is restricted to diffusion-driven processes (Parker et al., 2008; Rasa et al., 2011; Rolle et al., 2013a; Rolle and Kitanidis, 2014; Yang et al., 2017). To overcome these limitations, a series of technologies based on electrokinetic (EK) transport have been developed. Electrokinetic transport processes in porous media are generated by the application of an electric potential and include (Acar and Alshawabkeh, 1993; Reddy and Cameselle, 2009): (i) electromigration, consisting in the displacement of ions towards the electrodes of opposite charge, (ii) electroosmosis, which in fine sediments (e.g., silt and clay) results in a net movement of water as a consequence of the electrostatic charge surrounding solid grains, and (iii) electrophoresis, involving the displacement of charged particles.

Electrokinetic remediation (EKR) of contaminated porous media is based on the combination of multiple approaches that can involve the displacement of charged species via electromigration (Kim et al., 2009; Ribeiro and Mexia, 1997; Zhongming et al., 1997), the mobilization of non-charged compounds through electroosmosis (Schultz, 1997; Yustres et al., 2018), and the reactive degradation with oxidants or extracting agents delivered by electromigration (Gill et al., 2016; Pazos et al., 2008; Reynolds et al., 2008). Examples of reactive processes in electrokinetic systems include abiotic oxidation (Chowdhury et al., 2017; Fan et al., 2016; Wu et al., 2012a), biological degradation (Gill et al., 2014; Lohner et al., 2008a,b; Thevanayagam and Rishindran, 1998). EK technologies have the potential to target different types of contaminants including chlorinated ethenes (Hyldegaard et al., 2019; Mao et al., 2012; Ottosen et al., 2019), pesticides (López-Vizcaíno et al., 2017a,b; Risco et al., 2016), radionuclides (Jung et al., 2015; Kim et al., 2018) and heavy metals (Li et al., 1996; Masi et al., 2017a; Reddy et al., 2003; Virkutyte et al., 2002). Among the different electrokinetic 
mechanisms, electromigration is often the most important one and dominates the overall transport of charged species.

Despite electrokinetic remediation techniques have shown promising results in several laboratory experiments, the complexity and coupling of physical and chemical processes hinder a sound scientific understanding and entails difficulties in upscaling the outcomes of controlled laboratory tests to field scale engineering interventions at contaminated sites. Therefore, modeling of electrokinetic systems is an essential tool to integrate and quantitatively understand interlinked transport, electrostatic, and chemical processes in EKR systems. Modeling approaches with different levels of complexity have been proposed since the first studies on electrokinetics. Advanced codes for electrokinetic transport in porous media allow the description of reactive-transport of charged and non-charged compounds in 1-D and multidimensional domains (e.g. Appelo, 2017; Jacobs and Probstein, 1996; López-Vizcaíno et al., 2017c; Mao et al., 2019; Masi et al., 2017b; Mattson et al., 2002a,b; Paz-García et al., 2016; Rasouli, 2016; Sprocati et al., 2019; Wu et al., 2012b).

Although the underlying governing processes occurring during EK can be related with wellknown electrochemical systems, several aspects are often overlooked in environmental applications of electrokinetics. For instance, only a few formulations consider charge interactions between electrolyte ions by solving the Poisson-Nernst-Plank equations governing the displacement of ionic species (Newman and Thomas-Alyea, 2004; Pamukcu, 2009; PazGarcía et al., 2011; Sprocati et al., 2019). Furthermore, although reaction kinetics are known to be relevant in several situations in EK systems (Masi et al., 2019), their impact has not been systematically assessed. Finally, EKR experiments and models are typically performed in 1-D geometries without considering the transfer of the observed behavior and the effects on system performance in multidimensional setups. 
In this study we provide a detailed model-based assessment of transport and reactive processes in EK systems. We focus on electromigration transport in saturated porous media and we investigate: (i) Coulombic interactions and charge coupling between different ions in the pore water and their effects and limiting concentrations of a charged amendment that is delivered by EK as a function of the background electrolyte composition, (ii) the impact of compoundspecific diffusive properties and electrolyte mixtures, (iii) the effects of reaction kinetics on EK-based contaminant degradation, and (iv) the effect of dimensionality and electrode configurations when upscaling from 1-D laboratory to 2-D field-scale domains.

We present scenarios of electrokinetic conservative and reactive transport to illustrate the impact of the investigated mechanisms on macroscopic transport by EK and we use quantitative metrics to evaluate the difference between the conditions tested. The outcomes show that such mechanisms play a key role on the performances of EKR and should be taken into account for improved interpretation of laboratory experiments and for optimal design and operation of pilot and field-scale systems. 


\section{Modeling approach}

\subsection{Governing equations}

Coulombic interactions affect the displacement of charged species in porous media and need to be considered for both transport of charged solutes under natural conditions (Appelo and Wersin, 2007; Boudreau et al., 2004; Muniruzzaman et al., 2014; Muniruzzaman and Rolle, 2016; Rolle et al., 2013b) and for enhanced displacement resulting from the application of an electric field (Appelo, 2017; Krabbenhøft and Krabbenhøft, 2008; Newman and ThomasAlyea, 2004). In a saturated porous medium, under the assumption of negligible activity gradients, the total flux of a species $i$ depends on the effect of concentration, electric and hydraulic potential gradients, that result on diffusive/dispersive, migration, and advective fluxes according to the Nernst-Planck equation (Appelo, 2017; Newman and Thomas-Alyea, 2004; Rasouli et al., 2015):

$$
J_{i}^{T o t}=\underbrace{-n D_{i} \nabla c_{i}}_{J_{i}^{D i f}} \underbrace{-n D_{i} \frac{z_{i} F}{R T} c_{i} \nabla \Phi}_{J_{i}^{M i g}} \underbrace{+q^{*} c_{i}}_{J_{i}^{A} d v}
$$

where $n$ is the accessible porosity, $D_{i}$ is the pore diffusion/dispersion coefficient, which under the assumption of negligible mechanical dispersion, is calculated as $D_{i}=D_{i}^{a q} \tau$ with $D_{i}^{a q}$ being the aqueous diffusion coefficient and $\tau$ the tortuosity, $\nabla c_{i}$ is the concentration gradient, $z_{i}$ the charge of $i, F$ the Faraday constant, $R$ the gas constant, $T$ the temperature, $c_{i}$ the molar concentration, $\nabla \Phi$ the electric potential gradient and $q^{*}$ is the specific water flux.

Considering a material balance for the species $i$, the governing multicomponent ionic transport equation reads as:

$$
\frac{\partial\left(n c_{i}\right)}{\partial t}+\nabla \cdot \boldsymbol{J}_{i}^{T o t}=r_{i}
$$

where $r_{i}$ is the reactive source/sink term. 
The Poisson's equation defines the relation between electric field and electric charge density $\left(\rho_{e}\right)$ and can be written as (Newman and Thomas-Alyea, 2004; Sastre and Santaballa, 1989):

$$
\nabla^{2} \Phi=-\frac{F}{\varepsilon} \sum_{i=1}^{N} Z_{i} c_{i}=-\frac{\rho_{e}}{\varepsilon}
$$

in which $\varepsilon$ is the absolute permittivity of the medium and $N$ is the number of charged species in solution. At a continuum scale, Eq. (3) ensures the validity of the electroneutrality condition (MacGillivray, 1968; Sastre and Santaballa, 1989):

$$
\sum_{i=1}^{N} z_{i} c_{i}=0
$$

and the Laplace's equation for the potential:

$$
\nabla^{2} \Phi=0
$$

The set of Eq. (1), (2) and (3) represents the Nernst-Planck-Poisson equations (NPPE) governing the transport of charged species. These equations should be used to describe transport during EKR in saturated porous media.

In absence of an external electric field, Coulombic interactions affect the distribution and concentration of charged species both in diffusion-dominated systems (Alt-Epping et al., 2015; Appelo et al., 2010, 2008; Boudreau et al., 2004; Liu et al., 2011) and under advectiondominated flow-through conditions (Muniruzzaman and Rolle, 2017, 2015; Rolle et al., 2018, 2013b). Charge interactions become of fundamental importance for the transport of charged species when an electric field is applied resulting in a net current density in the porous medium $(\boldsymbol{I} \neq 0)$. In such conditions, electrostatic interactions need to be taken into account to reproduce solute behavior (Appelo, 2017; Krabbenhøft and Krabbenhøft, 2008).

Considering the dominant contribution of the conductivity in the pore water solution it is possible to express the current density as the sum of the fluxes of the different ionic species (Newman and Thomas-Alyea, 2004; Pamukcu, 2009): 


$$
\boldsymbol{I}=F \sum_{i=1}^{N} z_{i} \boldsymbol{J}_{i}^{T o t}
$$

Combining the expression of the current density with the expression of the Nernst-Planck fluxes (Eq. (1)):

$$
\boldsymbol{I}=-F \sum_{i=1}^{N} z_{i} n D_{i} \nabla c_{i}-\left(F^{2} \sum_{i=1}^{N} z_{i}^{2} \frac{n D_{i}}{R T} c_{i}\right) \nabla \Phi+F \boldsymbol{q}^{*} \sum_{i=1}^{N} z_{i} c_{i}
$$

It is thus possible to express the continuity condition in terms of charge:

$$
F \sum_{i=1}^{N} z_{i} \frac{\partial\left(n c_{i}\right)}{\partial t}+\nabla \cdot\left(F \sum_{i=1}^{N} z_{i} \boldsymbol{J}_{i}^{T o t}\right)=F \sum_{i=1}^{N} z_{i} r_{i}
$$

The NPPE represent a highly non-linear system of equations; although in some particular cases and under steady-state conditions analytical solutions have been developed (Krabbenhøft and Krabbenhøft, 2008), numerical solutions are typically required for most multicomponent transport problems. In this study we adopt a formulation based on NPPE and we perform numerical simulations based on the modeling framework described in Sprocati et al. (2019). The code, NP-Phreeqc-EK, is implemented by coupling a flow and transport simulator (COMSOL Multiphysics) with a geochemical code (PhreeqcRM, Parkhurst and Wissmeier, 2015) through a MATLAB LiveLink interface, which allows describing fluid flow, solute transport, Coulombic interactions and biogeochemical reactions in saturated porous media.

\subsection{Fluxes and limiting concentrations during electromigration}

During EK, electromigration causes ions of opposite charge to migrate in opposite directions. Therefore, to maintain charge balance in every point of the domain, charge interactions arise to ensure electroneutrality in the domain. The study of such effects can be carried out considering an ideal EK system as in Figure 1. The figure schematically depicts an EK setup in which a porous medium is in contact with two electrode reservoirs. Such reservoirs have a 
large volume that allows neglecting the effects of electrolysis reactions and considering constant concentration of the species at the boundaries. An electrolyte consisting of ions $A^{+}$ and $B^{-}$is present in the cathodic reservoir, whereas the ions $C^{+}$and $D^{-}$constitute the background electrolyte solution in the porous medium and in the anode reservoir. The purpose of the EK system is to deliver the species $B^{-}$in the porous medium. In the following, we demonstrate that under certain conditions, it is possible to calculate the limiting concentration of $B^{-}$that can be delivered in the porous medium.

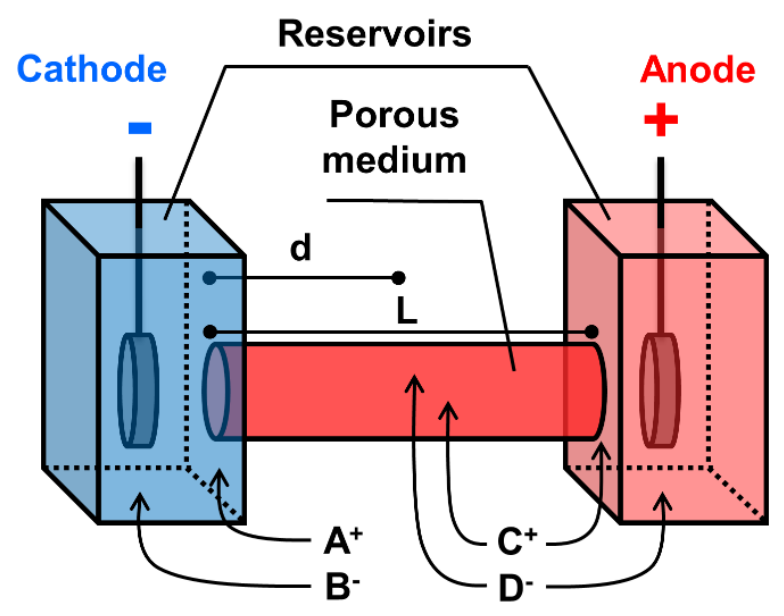

Figure 1. Schematic of the 1-D EK setup, with indication of the species in the ideal system at $t$ $=0$.

Assuming that electromigration is the major contributor to the current density, which is typically the case in dilute electrolyte systems (Newman and Thomas-Alyea, 2004), Eq. (7) can be simplified to:

$$
\boldsymbol{I}=F \sum_{i=1}^{N} J_{i}^{M i g} z_{i}=F^{2} \sum_{i=1}^{N} z_{i}^{2} \frac{n D_{i}}{R T} c_{i} \nabla \Phi=\sigma_{e} \nabla \Phi
$$

where $\sigma_{e}$ is the electrical conductivity of the electrolyte solution and $\boldsymbol{I}$ must be uniform over the length of the 1-D domain, in analogy to a circuit in series (Appelo, 2017). As a result of the electroneutrality condition, the concentration of cations must correspond to the concentration of anions in any point of the domain. In general, according to Eq. (9), if the diffusion 
coefficients or the valence of the different ions in the system differ, the current carried by anions can differ from the current carried by cations. However, assuming ideal ions with the same diffusion coefficient and valence, the current in the system carried by the anions must be equal to the current carried by the cations. Thus, the relative current density contribution of $B^{-}$ with respect to the total current can be written as:

$$
\begin{aligned}
& \frac{F Z_{B^{-}} J_{B^{-}}^{M i g}}{I}=\frac{z_{B^{-}} J_{B^{-}}^{M i g}}{z_{A^{+}} J_{A^{+}}^{M i g}+z_{B^{-}} J_{B^{-}}^{M i g}+z_{C^{+}} J_{C^{+}}^{M i g}+z_{D^{-}} J_{D^{-}}^{M i g}}= \\
& \quad=\frac{z_{B^{-}} J_{B^{-}}^{M i g}}{2\left(z_{B^{-}} J_{B^{-}}^{M i g}+z_{D^{-}} J_{D^{-}}^{M i g}\right)}=\frac{z_{B^{-}} J_{B^{-}}^{M i g}}{2\left(z_{A^{+}} J_{A^{+}}^{M i g}+z_{C^{+}} J_{C^{+}}^{M i g}\right)}
\end{aligned}
$$

It is possible to write the latter term of Eq. (10) for a state of the system in which, after a certain time, $B^{-}$has been delivered from the boundary at $x=0$ to a point in the domain at $x=d$ :

$$
\frac{z_{B^{-}} J_{B^{-}, x=d}^{M i g}}{\left(z_{A^{+}} J_{A^{+}, x=d}^{M i g}+z_{C^{+}} J_{C^{+}, x=d}^{M i g}\right)}=\frac{z_{B^{-}} J_{B^{-}, x=0}^{M i g}}{\left(z_{A^{+}} J_{A^{+}, x=0}^{M i g}+z_{C^{+}} J_{C^{+}, x=0}^{M i g}\right)}
$$

Assuming that $c_{A^{+}, x=d}=0$, since this cation cannot be transported in the domain by electromigration as the migration flux enforces its stalling at the cathode, Eq. (11) can be simplified as:

$$
\frac{Z_{B^{-}} J_{B^{-}, x=d}^{M i g}}{Z_{C^{+}} J_{C^{-}, x=d}^{M i g}}=\frac{z_{B^{-}} J_{B^{-}, x=0}^{M i g}}{z_{A^{+}} J_{A^{+}, x=0}^{M i g}+z_{C^{+}} J_{C^{+}, x=0}^{M i g}}
$$

Therefore, the flux of the delivered species $B^{-}$in the porous medium domain can be written as:

$$
Z_{B^{-}} J_{B^{-}, x=d}^{M i g}=\frac{Z_{B^{-}} J_{B^{-}, x=0^{M}}^{M i g} Z_{C^{+}} J_{C^{+}, x=d}^{M i g}}{Z_{A^{+}} J_{A^{+}, x=0}^{M i g}+Z_{C^{+}} J_{C^{+} x=0}^{M i g}}
$$

Since cations and anions in the system have the same valence and diffusion coefficient, they carry the same current. Given the electroneutrality condition, the conductivity of the electrolyte solution must be uniform, which in turn implies a uniform electric potential gradient in the 
domain. With these considerations it is possible to simplify Eq. (13), using the definition of the migration flux given in Eq. (1), to derive the concentration of $B^{-}$at distance $x=d$ as function of concentrations at the boundary and within the porous medium:

$$
c_{B^{-}, x=d}=\frac{c_{B^{-}, x=0} c_{C^{+}, x=d}}{c_{A^{+}, x=0}+c_{C^{+}, x=0}}
$$

A more general expression of (14) can be derived introducing the quantity $\Gamma$, accounting for the concentration of multiple ions in the system:

$$
\Gamma=\frac{1}{2} \sum_{i=1}^{N} c_{i}
$$

where $N$ is the total number of charged species. In the system described above and depicted in Figure $1, N$ is equal to $4, \Gamma_{x=0}=0.5\left(c_{A^{+}, x=0}+c_{B^{-}, x=0}+c_{C^{+}, x=0}+c_{D^{-}, x=0}\right)=c_{A^{+}, x=0}+$ $c_{C^{+}, x=0} \quad$ and $\quad \Gamma_{x=d}=0.5\left(c_{D^{-}, x=d}+c_{C^{+}, x=d}\right)=c_{C^{+}, x=d} \quad$ as $\quad$ a consequence of the electroneutrality condition. Thus, Eq. (14) can be generalized to:

$$
c_{B^{-}, x=d}=\frac{c_{B^{-}, x=0} \Gamma_{x=d}}{\Gamma_{x=0}}
$$

A similar expression has been recently proposed (Sprocati et al., 2019) as an empirical formulation for the maximum amendment concentration that can be delivered through electromigration, based on the analysis of previous experiments delivering permanganate and nitrate in porous media.

It is interesting to note that according to Eq. (16), when the electrolyte concentration at the boundary and within the domain are the same $\left(\Gamma_{x=0}=\Gamma_{x=d}\right)$, it is possible to deliver in the porous medium the same concentration of $B^{-}$present in the cathode reservoir. When the concentrations of background electrolyte ions in the pore water solution are smaller than at the boundary $\left(\Gamma_{x=d}<\Gamma_{x=0}\right)$, the concentration of $B^{-}$that can be delivered is limited and smaller than the value at the boundary. Conversely, in the case $\Gamma_{x=d}>\Gamma_{x=0}$, the concentration of $B^{-}$ in the porous medium can be higher than its value at the boundary. Moreover, in case the ratio 
$c_{B^{-}, x=0} / \Gamma_{x=0}$ decreases as a result of electrolysis reactions or addition of buffering electrolytes in the electrode reservoir, then $c_{B^{-}, x=d}$ can decrease significantly, leading to situations of stalling of the amendment delivery (Hodges et al., 2013).

In the following sections, these concepts are illustrated and tested with numerical simulations and the results are discussed in the context of charged species distribution in electrokinetic applications. 


\subsection{EK transport scenarios}

Transport by electromigration is critical for EKR of contaminated porous media. For instance, transport of negatively charged ions is of key importance when EK techniques are combined with in-situ chemical oxidation (EK-ISCO) or with enhanced biodegradation (EK-Bio). EKISCO approaches rely on the delivery of reactive anions such as permanganate or persulfate (e.g. Lima et al., 2017; Wu et al., 2013, 2012a,b) whereas EK-Bio techniques aims at distributing charged substrates and electron acceptors, such as lactate (Mao et al., 2012) and nitrate (Gill et al., 2015) to stimulate the degradation activities of indigenous bacteria.

In the following we illustrate selected scenarios highlighting the most relevant mechanisms affecting amendment delivery and contaminant degradation, their coupling and mutual interactions, and their effect on the performance of EKR systems.

In the first scenario, we perform conservative transport simulations in an ideal system of monovalent ions $\left(A^{+}, B^{-}, C^{+}\right.$and $\left.D^{-}\right)$with the same diffusion coefficients, analogous to the system depicted in Figure 1. At the beginning of the simulation, only $C^{+}$and $D^{-}$are present in the $1 \mathrm{~m}$ long porous medium domain, whereas $A^{+}$and $B^{-}$are present at the cathodic boundary. With the application of a voltage difference $(100 \mathrm{~V})$ at the electrodes, electromigration causes the displacement of the anion $B^{-}$from the boundary in the domain. We analyze the propagation of the $B^{-}$front in the porous medium and the impact of different electrolyte concentrations in the background and at the inlet boundary. We also compare the outcomes of the numerical simulations with the simple formula proposed and derived in the previous section (Eq. (16)) to estimate the amendment concentration.

The second scenario is analogous to the previous one but focuses on the migration of real ions and on the effect of compound-specific diffusive properties during EK-based amendment delivery. We consider transport of charged species used in EKR applications such as 
permanganate and lactate. First, we consider the presence at the cathode boundary of a solution of $\mathrm{K}^{+}$and $\mathrm{MnO}_{4}^{-}$, whereas, in a second case, the electrolyte present in the cathodic reservoir contains $\mathrm{K}^{+}$and $\mathrm{C}_{3} \mathrm{H}_{5} \mathrm{O}_{3}^{-}$. In both situations, an initial background solution of $\mathrm{HCO}_{3}^{-}$and $\mathrm{Na}^{+}$ is present throughout the porous medium domain.

In the third scenario we investigate reactive transport effects on EKR performance. We consider the same 1-D setup in which a charged species $\left(B^{-}\right)$is transported into the domain by electromigration and reacts with a non-charged organic contaminant (Org) present in the porous medium. In such conditions, the reactants come into contact through EK-controlled mixing, and the following reaction occurs:

$$
B^{-}+\operatorname{Org} \rightarrow D^{-}
$$

where the reaction product $D^{-}$ensures that the reaction does not produce any charge imbalance in the system. The rate of the bimolecular reaction is expressed as:

$$
\frac{d c_{B^{-}}}{d t}=-\lambda c_{O r g} c_{B^{-}}
$$

where $\lambda$ is the kinetic rate constant. In analogy to the first scenario, the reactive transport simulations are performed for the ideal system of monovalent ions with the same diffusion coefficient. Reactive transport is assessed in this test for three different concentrations of the background electrolyte ions $\left(C^{+}\right.$and $\left.D^{-}\right)$, highlighting the impact of Columbic interactions for the simulation of reactive processes during EKR.

In the fourth scenario, we explore the impact of reaction kinetics on EKR and we compare degradation efficiencies in a 1-D system with same geometry as in the previous examples. In these simulations, the electric field is turned off after a certain time, to evaluate the temporal evolution of the reaction between the delivered amendment $\left(B^{-}\right)$and the organic contaminant 
$(\mathrm{Org})$. The comparison is carried out considering the same electric potential field and investigating three different reaction kinetics in scenarios with different initial concentrations of the organic compound.

Finally, in the fifth scenario we investigate the effect of dimensionality on EK transport. We consider both conservative and reactive transport in 2-D field scale setups. Three different electrode configurations are simulated, in which a first configuration, Conf. 1, represents an ideal case of continuous linear arrays of electrodes that mimic ideal 1-D geometries used in laboratory experiments. The other two configurations, Conf. 2 and Conf. 3, are representative of field applications (Masi et al., 2017a) and electrodes are arranged with same spacing (1 m) but different polarity scheme. The goal of these simulations is to show that despite the use of a unique distance between electrodes of opposite polarities, large differences in the estimated performances can arise when considering multidimensional effects on EK transport and different electrode configurations.

An overview of the considered scenarios and their key features is presented in Table 1, whereas operational parameters common to all simulations and the diffusion coefficients of the different species are listed in Table 2. 
Table 1. Overview of the 1-D and 2-D tests with indication of the key features.

\begin{tabular}{cll}
\hline Case & \multicolumn{1}{c}{ Test } & \multicolumn{1}{c}{ Key features } \\
\hline 1 & 1-D conservative, ideal ions & $\begin{array}{l}\text { Conservative transport, equal diffusion coefficients, different } \\
\text { concentrations of background electrolyte }\end{array}$ \\
2 & 1-D conservative, real ions & $\begin{array}{l}\text { Conservative transport, } \mathrm{KMnO}_{4} \text { and } \mathrm{KC}_{3} \mathrm{H}_{5} \mathrm{O}_{3} \text { at the cathode, } \\
\text { different concentrations of background } \mathrm{NaHCO}_{3}\end{array}$ \\
3 & $\begin{array}{l}\text { 1-D reactive, effect of background } \\
\text { concentration }\end{array}$ & $\begin{array}{l}\text { Reactive transport, equal diffusion coefficients, fast kinetics, } \\
\text { different concentrations of background electrolyte }\end{array}$ \\
4 & $\begin{array}{l}\text { 1-D reactive, effect of reaction } \\
\text { kinetics and concentration of non- } \\
\text { charged reactant } \\
\text { 2-D electrode configurations for } \\
\text { conservative and reactive transport, } \\
\text { dimensionality effects }\end{array}$ & $\begin{array}{l}\text { Reactive transport, equal diffusion coefficients, different } \\
\text { reaction kinetics, different initial concentrations of the non- } \\
\text { charged reactant, equal concentration of background electrolyte } \\
\text { Conservative and reactive transport with one reaction kinetics, } \\
\text { equal diffusion coefficients, equal concentration of background } \\
\text { electrolyte, } 3 \text { different electrode configurations }\end{array}$ \\
\hline
\end{tabular}

Table 2. Parameters of EKR implemented in the different modeling scenarios and aqueous diffusion coefficients at $T=25^{\circ} \mathrm{C}$.

\begin{tabular}{|c|c|c|}
\hline Parameter & Unit & Value \\
\hline Distance between electrodes (L) & $\mathrm{m}$ & 1 \\
\hline Temperature & ${ }^{\circ} \mathrm{C}$ & 25 \\
\hline Porosity & - & 0.4 \\
\hline Tortuosity & - & 0.4 \\
\hline Electric potential at the Cathode $(x=0)$ & $\mathrm{V}$ & 0 \\
\hline Electric potential at the Anode $(\mathrm{x}=\mathrm{L})$ & $\mathrm{V}$ & 100 \\
\hline Species & & $D^{a q}\left[\mathrm{~m}^{2} / \mathrm{s}\right]$ \\
\hline $\mathrm{A}^{+}, \mathrm{B}^{-}, \mathrm{C}^{+}, \mathrm{D}^{-}$ & & $1.00 \times 10^{-9}$ \\
\hline $\mathrm{K}^{+}$ & & $1.96 \times 10^{-9}$ \\
\hline $\mathrm{MnO}_{4}^{-}$ & & $1.63 \times 10^{-9}$ \\
\hline $\mathrm{C}_{3} \mathrm{H}_{5} \mathrm{O}_{3}{ }^{-}$ & & $1.03 \times 10^{-9}$ \\
\hline $\mathrm{Na}^{+}$ & & $1.33 \times 10^{-9}$ \\
\hline $\mathrm{HCO}_{3}^{-}$ & & $1.18 \times 10^{-9}$ \\
\hline
\end{tabular}




\section{Results and discussion}

\subsection{1-D conservative, ideal ions}

This scenario considers the conservative displacement of an ideal anion, $B^{-}$, from the cathode $(x=0 \mathrm{~m})$ towards the anode $(x=1 \mathrm{~m})$. The initial concentrations of both $B^{-}$and $A^{+}$at the cathodic boundary are equal to $10 \mathrm{mM}$ in all simulations (Table 3 ) whereas the concentrations of the ions in the background electrolyte $\left(C^{+}\right.$and $\left.D^{-}\right)$are different. In the following we refer to $\Gamma_{\text {low }}, \Gamma_{\text {equal }}$ and $\Gamma_{\text {high }}$ to indicate the considered cases with different background electrolyte concentration in the porous medium. Specifically, "low", "equal", and "high" refer to the concentration of the background electrolyte $\left(C^{+}\right.$and $\left.D^{-}\right)$in the domain with respect to the concentration of $A^{+}$and $B^{-}$at the cathodic boundary. In addition, scenario $\Gamma_{\text {inflow }}$ represents the case where at the cathodic boundary all ions, including $C^{+}$and $D^{-}$, are present at a concentration of $10 \mathrm{mM}$. This last case is considered of particular relevance for practical applications as $\Gamma$ tends to rise in electrode wells if recirculation is not performed due to electrolysis reactions and/or addition of buffer solutions.

Table 3. Summary of background concentrations and concentrations at the boundary $(x=0)$ for a system of ideal ions.

\begin{tabular}{|c|c|c|c|c|c|}
\hline \multirow[b]{2}{*}{ Species } & \multirow[b]{2}{*}{ Unit } & \multicolumn{4}{|c|}{ Concentration in the domain } \\
\hline & & $\Gamma_{\text {low }}$ & $\Gamma_{\text {equal }}$ & $\Gamma_{\text {high }}$ & $\Gamma_{\text {inflow }}$ \\
\hline $\mathrm{A}^{+}$ & $\mathrm{mM}$ & 0 & 0 & 0 & 0 \\
\hline $\mathrm{B}^{-}$ & $\mathrm{mM}$ & 0 & 0 & 0 & 0 \\
\hline $\mathrm{C}^{+}$ & $\mathrm{mM}$ & 2.5 & 10 & 40 & 10 \\
\hline $\mathrm{D}^{-}$ & $\mathrm{mM}$ & 2.5 & 10 & 40 & 10 \\
\hline \multirow[b]{2}{*}{ Species } & & \multicolumn{4}{|c|}{ Concentration at the cathode boundary $x=0$} \\
\hline & Unit & $\Gamma_{\text {low }}$ & $\Gamma_{\text {equal }}$ & $\Gamma_{\text {high }}$ & $\Gamma_{\text {inflow }}$ \\
\hline $\mathrm{A}^{+}$ & $\mathrm{mM}$ & 10 & 10 & 10 & 10 \\
\hline $\mathrm{B}^{-}$ & $\mathrm{mM}$ & 10 & 10 & 10 & 10 \\
\hline $\mathrm{C}^{+}$ & $\mathrm{mM}$ & 0 & 0 & 0 & 10 \\
\hline $\mathrm{D}^{-}$ & $\mathrm{mM}$ & 0 & 0 & 0 & 10 \\
\hline
\end{tabular}



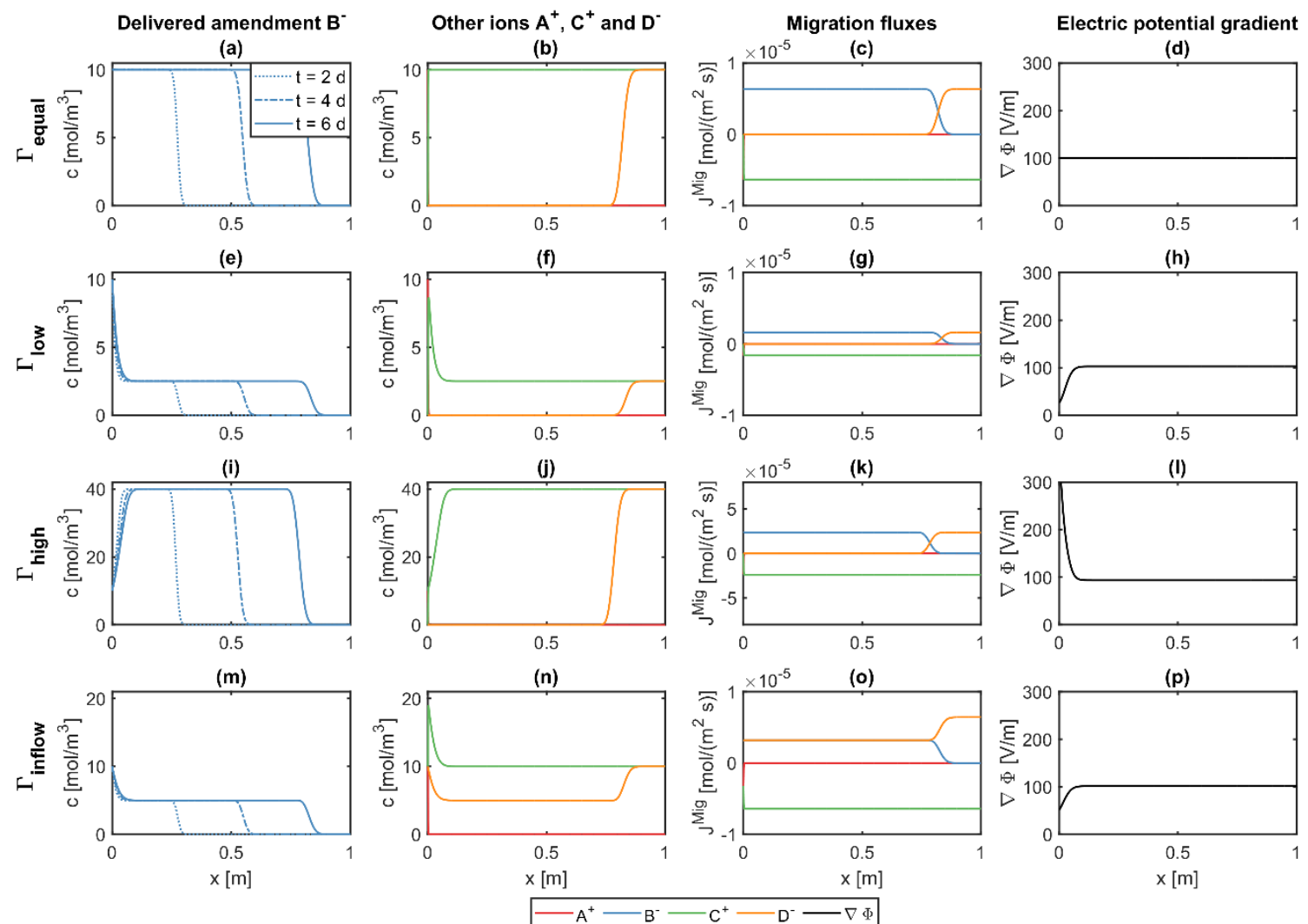

Figure 2. Simulated concentration profiles of $B^{-}$delivered by electromigration for cases "equal", "low", "high" and "inflow". In the first column, the lines show concentration profiles of $B^{-}$at $t=2,4$ and 6 days whereas in the other columns the concentration profiles of the background ions $C^{+}$and $D^{-}$, the migration fluxes of the species in the system, and the resulting electric potential gradients are shown at $t=6$ days.

Figure 2 shows that for the case $\Gamma_{\text {equal }}$, the concentration of $B^{-}$in the domain is equal to the one at the boundary $(10 \mathrm{mM}$, Figure $2 \mathrm{a})$. However, for both scenarios $\Gamma_{\text {low }}$ and $\Gamma_{\text {high }}$, the concentration of $B^{-}$appears to reach considerably lower (Figure 2e) and higher (Figure 2i) values with respect to the concentration in the reservoir. In such cases, the concentration of $B^{-}$is equal to the concentration of the background ions (either $C^{+}$or $D^{-}$), which is also equal to the term $\Gamma$. These results of the NPPE numerical simulations agree with the values that can be calculated with the formula presented in Eq. (16). The proposed equation allows a correct 
estimation of $B^{-}$in the domain also for the case $\Gamma_{\text {inflow }}$ for which, despite the background concentration of ions in the domain is the same as for the case $\Gamma_{\text {equal }}$, the higher $\Gamma$ at the boundary makes $c_{B^{-}, x=d}<c_{B^{-}, x=0}$.

Besides the invading front of $B^{-}$in the porous medium, electromigration also entails the coupled movement of the electrolyte background ions: $C^{+}$is displaced towards the cathode, whereas $D^{-}$moves towards the anode correspondingly to the invading front of $B^{-}$(Figure $2 \mathrm{~b}$, $f, j$ and $n$ ). For an explanation of these results, it is necessary to consider the mechanisms of electromigration transport and the charge interactions between the different ions. Due to the electroneutrality condition, the concentration of cations and anions must be equal throughout the domain. Consequently, having considered all ions in the system with same valence and diffusion coefficient, also the migration fluxes (thus the contribution of the different ions to the current density) of anions and cations must be equal (Figure 2c, g, k and o). Ultimately, it is the balance of the migration fluxes between the invading anion and the resident cation in the porous medium that causes the change in concentration of $B^{-}$in the domain compared to its value at the boundary.

For scenario $\Gamma_{\text {inflow }}$, being $D^{-}$present at the electrode reservoir and migrating in the same direction as $B^{-}$, the contribution to the current of the anions is provided by both $D^{-}$and $B^{-}$. As a result, the concentration of $B^{-}$in the domain is lower in $\Gamma_{\text {inflow }}$ with respect to $\Gamma_{\text {equal }}$, as the required contribution to the current to be supplied by the anions also considers the contribution of $D^{-}$.

This scenario highlights that an estimation of $\Gamma$ both at the boundary and in the system is critical to predict the expected concentration of a charged solute that is delivered by electromigration from an electrode reservoir into a porous medium domain. Moreover, the outcomes of the simulations show that the concentration of species in the electrode reservoirs should be controlled to maximize the delivered concentration of the charged species of interest 
in the domain. For practical applications, the latter observation suggests that, in order to maximize the concentration of a species that can migrate from a boundary into the domain, such species should be added to a dilute solution at the boundary reservoir. Alternatively, the species should be supplied in great excess if other charged solutes are present at significant concentrations in the reservoir solution.

\subsection{1-D conservative, real ions}

In this scenario, the same geometry and delivery approach of the previous test is adopted. We consider two cases with the delivery of a permanganate $\left(\mathrm{MnO}_{4}^{-}\right)$and a lactate $\left(\mathrm{C}_{3} \mathrm{H}_{5} \mathrm{O}_{3}^{-}\right)$ solutions, respectively. In both examples, the same background electrolyte consisting of $\mathrm{HCO}_{3}^{-}$ and $\mathrm{Na}^{+}$ions was present in the porous medium. The concentration of the ions delivered from the cathode and that of the ions present in the domain correspond to the scenario $\Gamma_{\text {equal }}$ discussed in the previous section. In these simulations, the focus is on the charge interactions and the effects of compounds specific properties for real ions with different diffusion coefficients.

In the first case, $\mathrm{MnO}_{4}^{-}\left(D^{a q}=1.63 \times 10^{-9} \mathrm{~m}^{2} / \mathrm{s}\right)$ is delivered from the cathodic reservoir and migrates in the domain replacing the background anion $\mathrm{HCO}_{3}^{-}\left(D^{a q}=1.18 \times 10^{-9} \mathrm{~m}^{2} / \mathrm{s}\right)$. The results show that the advancing front of permanganate in the domain is significantly spread and reaches concentrations higher than $10 \mathrm{mM}$, corresponding to the concentration of $\Gamma$ both at the inflow and in the background (Figure 3a). Similarly, also the background ions show spread profiles and an accumulation of sodium occurs in the first part of the domain. The analysis of the migration fluxes helps interpreting the observed behavior. As the diffusive properties of the solutes are different, the contributions to the current density of anions and cations are distinct. Moreover, as different anions are present in the system (i.e., the delivered anion and the background anion, $\mathrm{HCO}_{3}^{-}$), their proportion to the current density is also dissimilar. For the 
same concentration of $\mathrm{HCO}_{3}^{-}$in the background and of permanganate delivered from the reservoir, $\mathrm{MnO}_{4}^{-}$has higher diffusivity and, thus, provides a larger migration flux contribution than $\mathrm{HCO}_{3}^{-}$(Figure 3c).
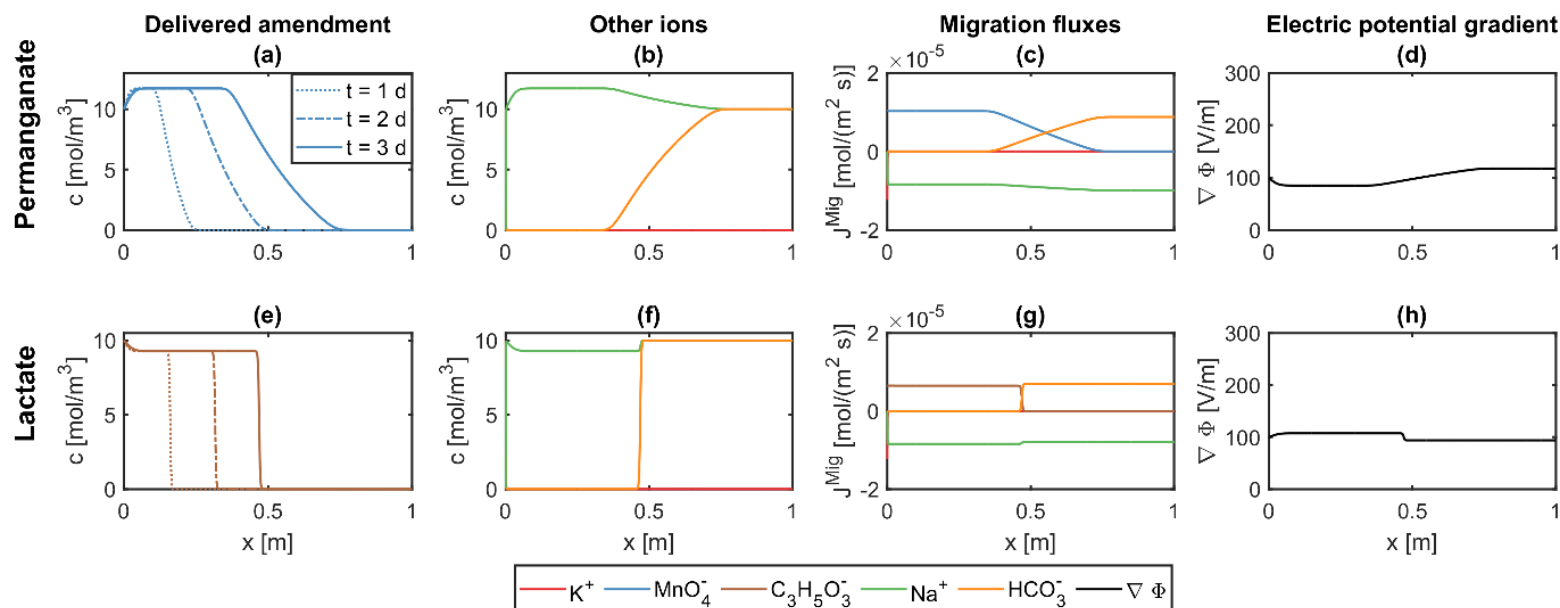

Figure 3. Simulated concentration profiles for the delivery of permanganate and lactate at different times ( $a$ and $e$ ). In both cases the remaining panels show the concentration profiles of the background ions $\mathrm{Na}^{+}$and $\mathrm{HCO}_{3}{ }^{-}$, the migration fluxes, and the electric potential gradient at $t=3$ days.

As the current must be uniform along the domain, in response to an increase in the contribution to the current density of the anions $\left(\mathrm{MnO}_{4}^{-}\right.$from the boundary), a decrease of the migration flux of the cations must follow. The latter term is only provided by $\mathrm{Na}^{+}$, thus the magnitude of the migration flux of this ion decreases close to the cathodic boundary (Figure 3c). Since $\mathrm{Na}^{+}$is transported from right to left, a decrease in magnitude in the migration flux results in an increase in its concentration in the left portion of the domain, which ultimately leads to an increase in the concentration of $\mathrm{MnO}_{4}^{-}$to maintain electroneutrality. Such phenomenon is also directly related with the spatial profile of the electric potential gradient. In contrast to the scenario with ideal ions, now $\nabla \Phi$ assumes different values depending on the electric 
conductivity in different parts of the domain, and ultimately contributes to the values of the migration fluxes with a mutual interplay.

In the case of lactate delivery, $\mathrm{C}_{3} \mathrm{H}_{5} \mathrm{O}_{3}^{-}\left(D^{a q}=1.03 \times 10^{-9} \mathrm{~m}^{2} / \mathrm{s}\right)$ has a lower diffusion coefficient than $\mathrm{HCO}_{3}^{-}$, which results in a very different behavior compared to the delivery of permanganate. A sharp front characterizes the migration of lactate in the domain and the plateau concentration reached is now lower that $10 \mathrm{mM}$ (Figure 3e). The lower contribution to the total current of the delivered anion causes the migration flux of $\mathrm{Na}^{+}$to increase in magnitude in proximity of the cathodic reservoir, with a reduced value of $\Gamma$ in the same zone (Figure $3 \mathrm{f}$ and $\mathrm{g}$ ).

The differences in the migration velocities of the different ions also cause different breakthrough behaviors. Figure 4 shows breakthrough curves of the delivered anions at the right boundary of the domain. The three lines represent the breakthrough of the ideal ion $B^{-}$ (in the case $\Gamma_{\text {equal }}$ of the previous scenario), permanganate, and lactate and show different behavior and maximum concentrations. In particular, only $B^{-}$reaches a concentration of 10 $\mathrm{mM}$ corresponding to the value in the inlet reservoir, whereas permanganate reaches a higher concentration $(11.71 \mathrm{mM})$ and lactate is limited to $9.28 \mathrm{mM}$. It can be observed that $\mathrm{MnO}_{4}^{-}$is the first ion that reaches the outer boundary due to its larger diffusion coefficient causing a more spread front in the porous medium (Figure 3a). The reason of the more spread shape of the spatial and breakthrough profiles is that when the front of $\mathrm{MnO}_{4}^{-}$migrates into the domain, its velocity is higher than the one of $\mathrm{HCO}_{3}^{-}$that is displaced in the same direction. As a result, a gradual transition phase develops at the $\mathrm{MnO}_{4}^{-}$front, which shows an elongated and less steep pattern. The opposite situation occurs for lactate, which has a smaller diffusion coefficient than $\mathrm{HCO}_{3}^{-}$that results in the steepest breakthrough curve. In fact, $\mathrm{HCO}_{3}^{-}$leaves the system with a migration velocity that is larger with respect to $\mathrm{C}_{3} \mathrm{H}_{5} \mathrm{O}_{3}^{-}$, with the macroscopic effect of steepening the lactate front that becomes similar to a shock wave. 


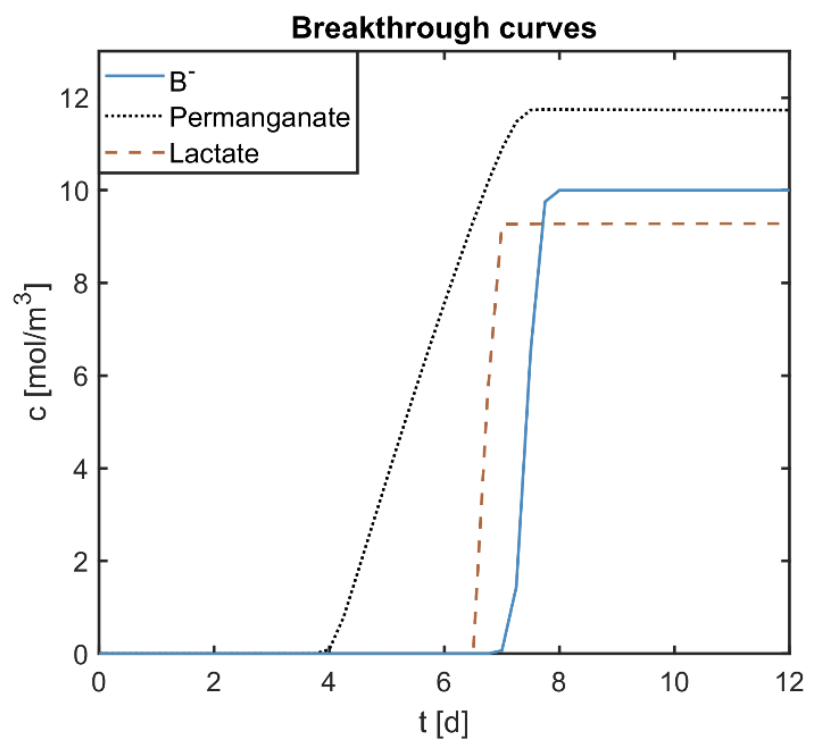

Figure 4. Breakthrough curves at the outlet boundary for the ideal ion $\mathrm{B}^{-}$, permanganate and lactate.

\subsection{1-D reactive, effect of background concentration}

In this scenario we consider reactive transport and the effects of Coulombic interactions on reactions occurring when a charged species delivered by electromigration comes into contact with a non-charged compound initially present at uniform concentration in the domain. The reaction stoichiometry and the kinetics are described by Eq. (17) and Eq. (18). In particular, we focus on the effect of different $\Gamma$ in the background pore water solution.

For natural transport in porous media, the reaction between the organic contaminant, Org, and the injected reactant, $B^{-}$, occurs only in the zones where diffusive/dispersive mixing between the reactants takes place (Hochstetler et al., 2013; Hochstetler and Kitanidis, 2013). However, during EK, the charged species $B^{-}$moves by electromigration and can overlap with the noncharged immobile compound. The EK-mixing can occupy the whole portion of the domain where the charged ion has migrated. This mixing phenomenon, typical of EK systems, provides 
an effective mixing mechanism in porous media between ionic and non-charged species. Such mixing mechanism is not restricted to thin zones with steep concentration gradients but relies on the relative movement of a reactant over the other and can stretch for large portions of the domain.
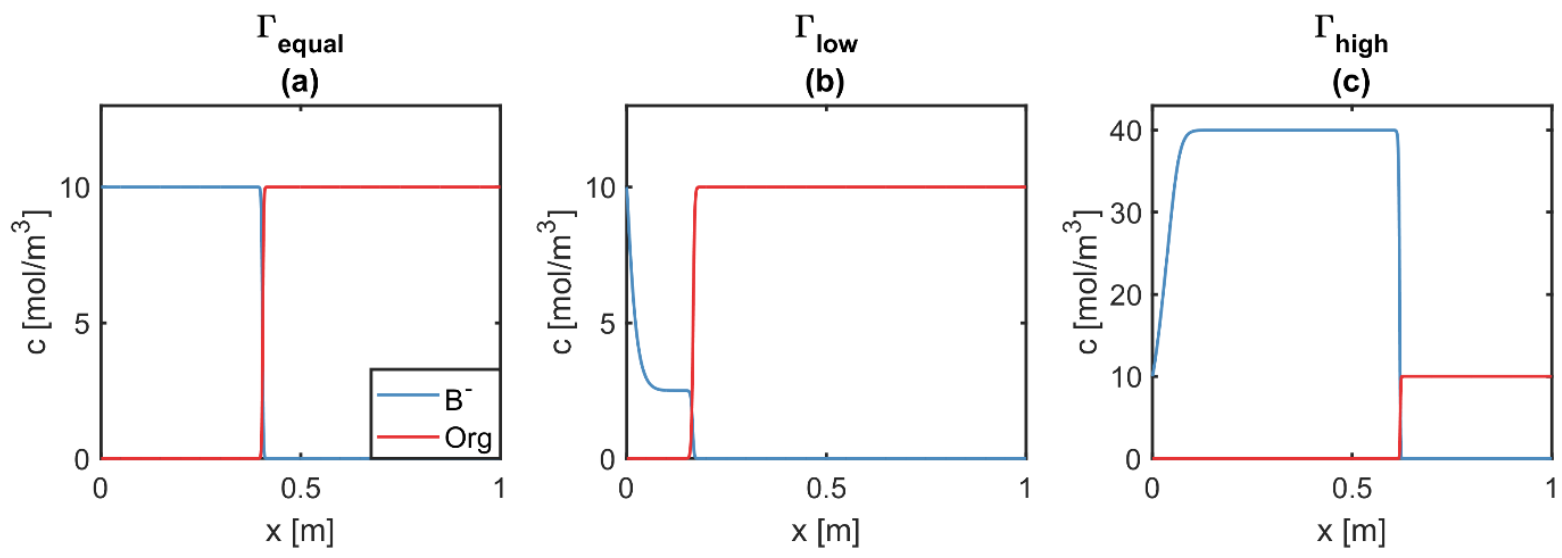

Figure 5. Simulated concentration profiles of $B^{-}$and Org after 6 days from the start of the simulation for scenarios with different concentration of background electrolyte ions: (a) "equal" (a), “low" (b), and "high” (c).

Figure 5 shows the effects of EK-induced reactions when $B^{-}$with the same concentration of $10 \mathrm{mM}$ at the cathodic reservoir is delivered in systems with different $\Gamma$ in the porous medium domain (10, 2.5, and $40 \mathrm{mM}$ for $\Gamma_{\text {equal }}, \Gamma_{\text {low }}$, and $\Gamma_{\text {high }}$, respectively). The kinetic rate constant for all cases is $\lambda=1 \times 10^{-4} \mathrm{~m}^{3} /(\mathrm{mol} \mathrm{s})$. Although the initial concentration of $\operatorname{Org}$ is the same for all scenarios, $\Gamma$ of the background plays a key role in the overall mass removal and in the propagation of the front of $B^{-}$in the porous medium. When $\Gamma$ in the background solution is smaller than the concentration of $B^{-}$at the boundary, the removal of the organic contaminant is the lowest (Figure 5b). In such case, according to Eq. (16), the concentration of $B^{-}$is limited by the value of $\Gamma$ and the removal of $\mathrm{Org}$ is reduced due to the small concentration of $B^{-}$that is brought into contact by electromigration and that can react with Org. This also corresponds 
to the apparent slower migration of the reactive front that, after 6 days, has only reached a distance of $0.2 \mathrm{~m}$. Conversely, when $\Gamma$ is larger than $B^{-}$at the cathodic reservoir, the concentration of $B^{-}$delivered by electromigration is higher and leads to enhanced consumption of $\mathrm{Org}$ and to a faster migration of the reactive front, arriving at $x=0.6 \mathrm{~m}$ in the same simulation time (Figure 5c). These results show the importance of the correct estimation of the delivered concentration of charged amendments: in practical applications of EK reactive transport, the concentration of $B^{-}$can significantly affect the intensity and duration of the applied electric field necessary to achieve the desired removal of the contaminants of concern.

\subsection{1-D reactive, effect of reaction kinetics}

In EK systems in which reactions can occur between a charged species mobilized by electromigration and non-charged compounds, important considerations involve the evolution of the system with respect to the stoichiometry and the rates at which such reactions proceed. Different behaviors are expected considering either fast or slow kinetics with respect to the velocity at which electromigration mixes the reactants.

The proposed examples consider cases in which $\Gamma$ in the background is equal to the concentration of $B^{-}$at the boundary and three different kinetic rate constants are considered $(\lambda$ $=1 \times 10^{-4}, 1 \times 10^{-6}$ and $\left.1 \times 10^{-7} \mathrm{~m}^{3} /(\mathrm{mol} \mathrm{s})\right)$. The simulations are also performed in systems with different initial concentrations of the organic compound. In these scenarios, the electric field is applied only for the first 6 days $\left(t_{E K}\right)$ and, afterwards, the system is allowed to react for 54 days. Such approach has been selected to evaluate the response of the system after the electric field is turned off, simulating a remediation strategy in which EK is only active to deliver the reactants in the contaminated area followed by a phase in which reactions can occur and no electric power is required. 

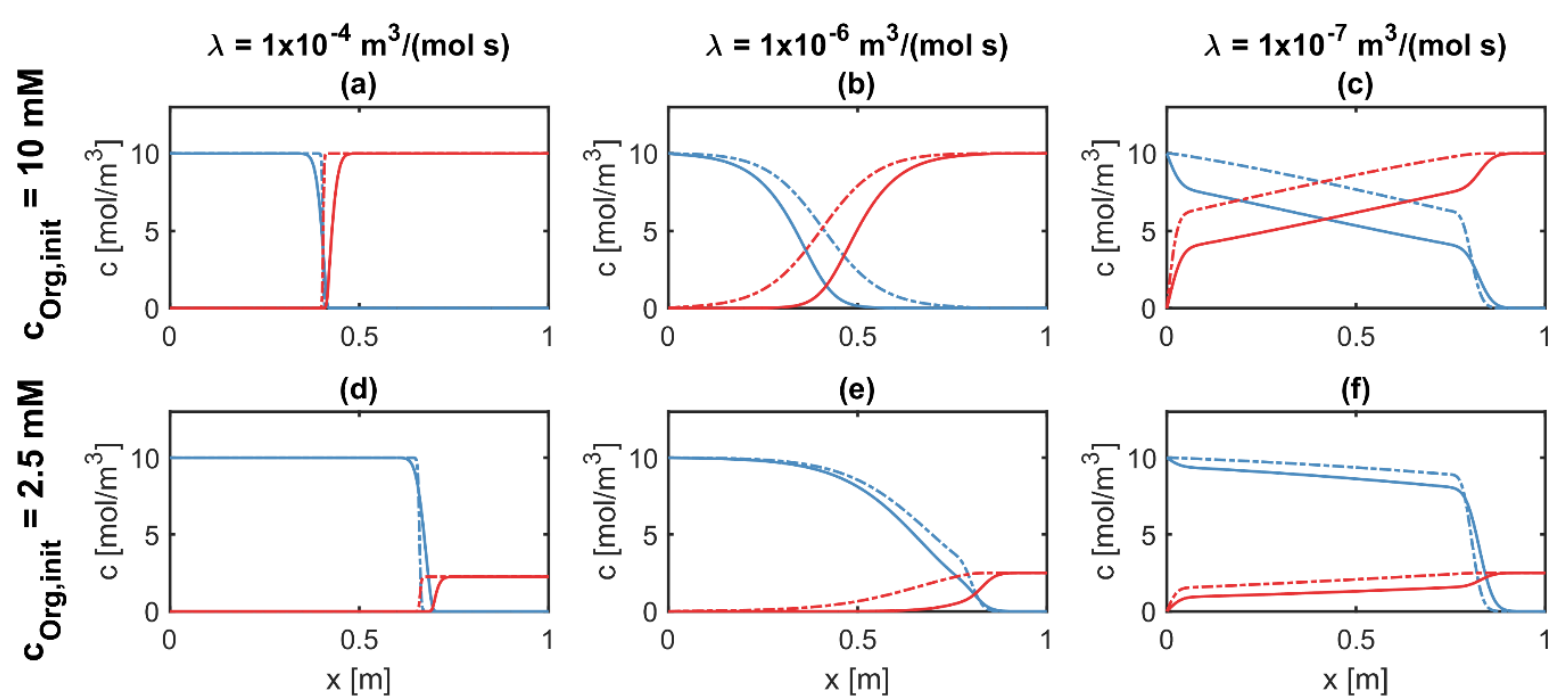

(e)
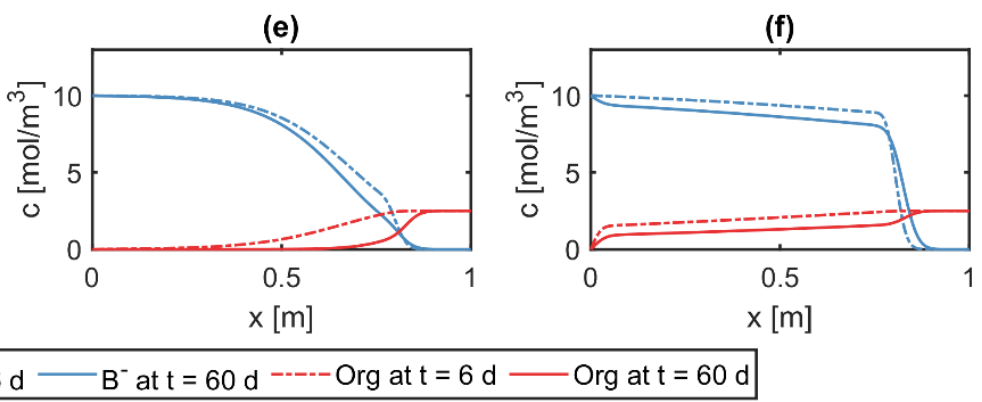

Figure 6. Simulated concentrations of $B^{-}$and Org after $6 d$ and $60 d$ for systems with kinetic rate constants of $\lambda=1 \times 10^{-4}, 1 \times 10^{-6}$ and $1 \times 10^{-7} \mathrm{~m}^{3} /(\mathrm{mol} \mathrm{s})$. The initial concentration of the non-charged organic compound is $10 \mathrm{~mol} / \mathrm{m}^{3}$ ( $a, b$ and c) and $2.5 \mathrm{~mol} / \mathrm{m}^{3}$ (d, e and f).

For cases in which the reactants are present at the same concentration in the domain, Figure 6a shows that for fast kinetic reactions the delivered reactant $\left(B^{-}\right)$quickly consumes the noncharged contaminant $(\mathrm{Org})$ and steep concentration gradients occur at the reactive front. Considering a slower reaction rate, Figure $6 \mathrm{~b}$ illustrates that an area with the simultaneous presence of $B^{-}$and $\mathrm{Org}$ occurs at the end of the active EK phase; the overlap of reactants concentrations ensures that at the reaction occurs also at later times. A similar outcome is achieved for the slowest kinetics (Figure 6c), where the mixing area affects a large portion of the domain.

For the cases in which the delivered reactant has higher concentrations than the non-charged contaminant (Figure 6d-f), the fronts of $B^{-}$have migrated farther compared to the respective cases with higher concentration of $\mathrm{Org}$. When the kinetics is fast, the shape of the profiles has not changed significantly and still shows steep concentration gradients (Figure 6d). In the case of slower kinetics (Figure 6e and f) a lower concentration of $\mathrm{Org}$ allows $B^{-}$to be delivered 
more effectively in the domain. In such conditions, however, the dependence of the kinetics (Eq. (18)) on the concentration of both reactants implies that the reaction rate at the end of the EK period is reduced and a smaller amount of the organic compound can be degraded over time.

To provide a quantitative comparison between the considered cases, we compute the relative mass $(R M)$ of the organic contaminant in the system:

$$
R M_{O r g}(t)=\frac{1}{M_{O r g, t=0}} \int_{\Omega} c_{O r g}(x, t) d \Omega
$$

where $M_{\text {org }, t=0}$ is the initial mass of the compound and $\Omega$ is the domain of interest.
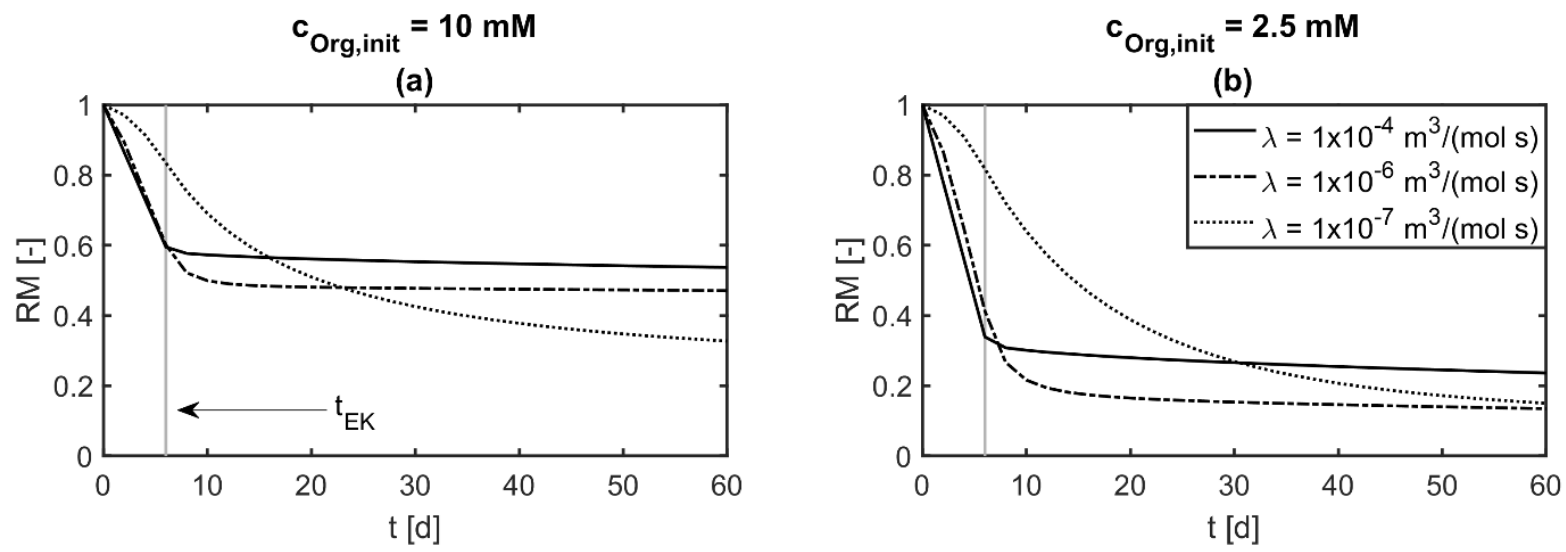

Figure 7. Relative mass of organic contaminant in the domain for reactive simulations performed with different kinetic constants. The vertical line represents the time when the electric field has been turned off $\left(t_{E K}=6\right.$ days $)$.

Figure 7 shows that higher removal rates can be achieved for systems where the non-charged compound is present at a lower concentration than the charged reactant delivered (Figure $7 \mathrm{~b}$ ). In such cases, the reactant transported by electromigration is in excess and the reactive front in the porous medium can advance faster. For both concentration levels of the non-charged compound, faster kinetics allow achieving higher contaminant removal during the initial phase 
of active application of the electric field. However, considering also the successive phase when the EK system is turned off, it is important to note that slower kinetics $\left(\lambda=1 \times 10^{-7}\right.$ and $\lambda=$ $\left.1 \times 10^{-6} \mathrm{~m}^{3} /(\mathrm{mol} \mathrm{s})\right)$ result in a higher contaminant removal with respect to the fast kinetics $(\lambda=$ $1 \times 10^{-4}$ and $\left.\mathrm{m}^{3} /(\mathrm{mol} \mathrm{s})\right)$. This is due to the fact that when the reactions occur slowly, large mixing zones are formed and the degradation in these zones can effectively proceed when EK is turned off. Considering the timeframe of the simulations performed, the slowest kinetics $(\lambda$ $=1 \times 10^{-7}$ and $\left.\mathrm{m}^{3} /(\mathrm{mol} \mathrm{s})\right)$ provides the highest removal, especially for the case with higher initial contaminant concentration. This is due to the overlapping of reactants occurring in large mixing zones.

These simulations show the importance of reaction kinetics in EK-driven contaminant removal and suggest that joint considerations of reaction kinetics and reactant concentrations need to be taken into account to select the optimal strategy of reactant delivery.

\subsection{2-D electrode configurations for conservative and reactive transport, dimensionality} effects

In this section we extend the considerations on electromigration-based delivery of charged species for conservative and reactive scenarios in multiple dimensions. So far, our considerations have been based on 1-D geometries typical of laboratory experiments. In such conditions, the current density is uniform throughout the domain. However, when upscaling EK applications to field pilot or full-scale systems, arrays of electrodes are used, which create spatially variable distributions of the current density and electric potential gradients and require multidimensional descriptions. In such setups, the delivery of species by electromigration, which is directly related to the electric potential gradients, becomes a function of the electrode configuration. In this scenario we performed simulations in a 2-D domain with three different electrode configurations. The first configuration (Conf. 1) is the ideal case in which anodes and 
cathodes are closely aligned along straight lines (Figure 8a) (Alshawabkeh et al., 1999; Kim et al., 2012). This layout is the only one directly comparable to 1-D geometries. However, electrode configurations in practical applications are different and layouts similar to the one represented in Figure 8d (Conf. 2) are typically adopted. In such configuration electrode wells are placed along an equally spaced regular grid, alternating rows of cathodes and anodes. The last configuration considered (Conf. 3) is analogous to Conf. 2 but the polarity of the electrodes is alternated in every column. To facilitate the comparison with the previous scenarios analyzed for 1-D geometries, the distance between electrodes of opposite polarity is set to1 m in all configurations, the radius of the electrode wells is set to $0.05 \mathrm{~m}$, and systems with ideal ions $\left(A^{+}, B^{-}, C^{+}\right.$and $\left.D^{-}\right)$and equal concentrations in the background and cathodic reservoir solutions $\left(\Gamma_{\text {equal }}\right)$ are considered.
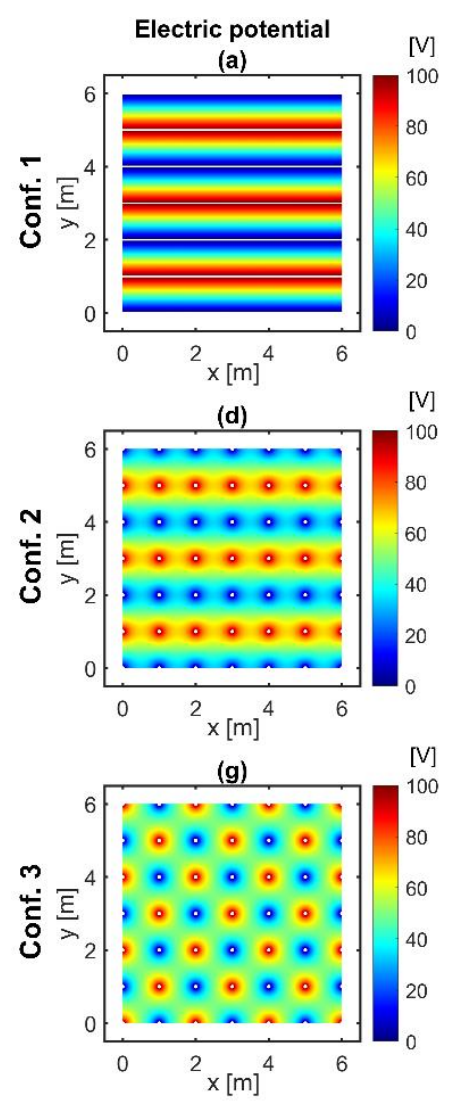
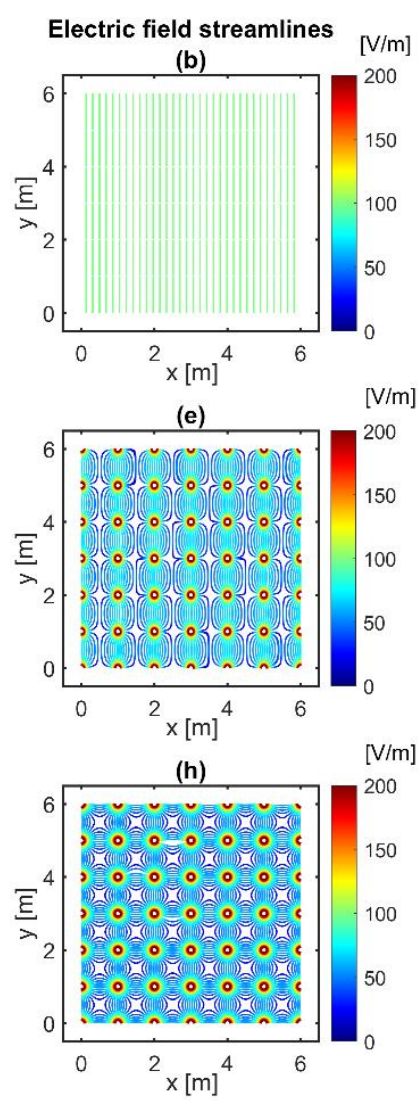
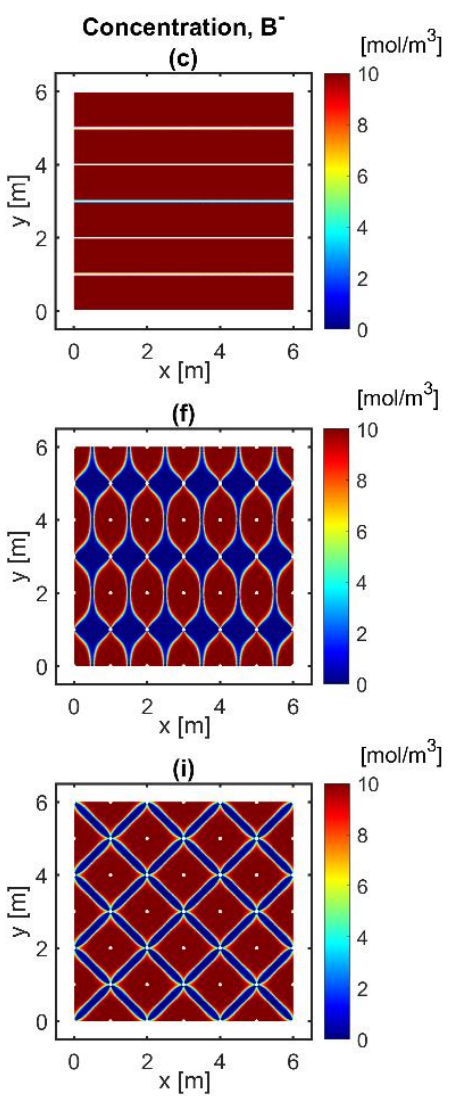

Figure 8. Simulated electric potential, streamlines, and spatial distribution of the delivered amendment $B^{-}$for different $2-D$ configurations at $t=8$ days. 
The computed distribution of electric potential and streamline patterns are different in the three configurations (Figure 8). Linear streamlines are obtained in Conf. 1 where the electrode layout is equivalent to a 1-D system (Figure $8 \mathrm{~b}$ ). In such setup the reactant $B^{-}$reaches the anode after 8 days $\left(t_{B T}\right)$ and its concentration is uniformly distributed throughout the 2-D domain.

In Conf. 2 the computed streamlines have the pattern illustrated in Figure 8e and, after the same simulation time, the breakthrough of $B^{-}$occurs at the anodes although its spatial delivery only occurs in a portion of the 2-D domain (Figure 8f). In other parts of the domain the delivered concentration is very low, particularly between the electrodes of equal polarity where electric field streamlines diverge. Similarly, in Conf. 3 the breakthrough of $B^{-}$at the anodes occurs after the same time but the distribution of this charged species in the 2-D domain appears to be more uniform (Figure 8i) than in Conf. 2.

To compare the performances of the different configurations, we compute the relative area $(R A)$ occupied by the delivered substrate, $B^{-}$:

$$
R A_{B^{-}, \text {lim }}(t)=\frac{1}{\Omega} \int_{\Omega}\left\{\begin{array}{ccc}
1 & \text { if } & c_{B^{-}}(\boldsymbol{x}, t) \geq c_{B^{-}, \text {lim }} \\
0 & \text { if } & c_{B^{-}}(\boldsymbol{x}, t)<c_{B^{-}, \text {lim }}
\end{array} d \Omega\right.
$$

where $c_{B^{-}, \text {lim }}$ is a threshold concentration that was set to $5 \mathrm{mM}$ in this example.

The delivery efficiency in the three considered configurations has been evaluated in Figure 9a, which shows the temporal evolution of the relative area. As expected, the ideal Conf. 1 provides a uniform distribution of $B^{-}$throughout the domain after $t_{B T}$. At the same time the $R A_{B^{-}, 5 m M}$ is 0.60 and 0.70 in Conf. 2 and Conf. 3, respectively. As a result, if the successful implementation of EK requires uniform distribution of a charged species throughout the domain, it would be necessary to apply the electric field for 28 days with Conf. 2 and for 17 days with Conf. 3. 
Conservative

(a)

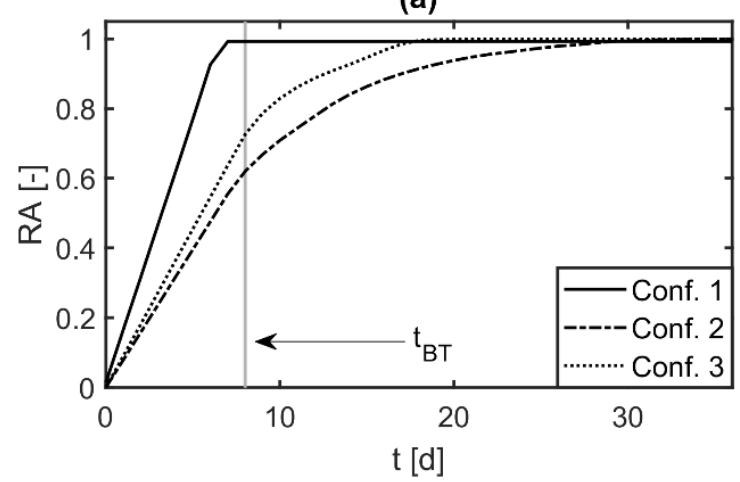

Reactive

(b)

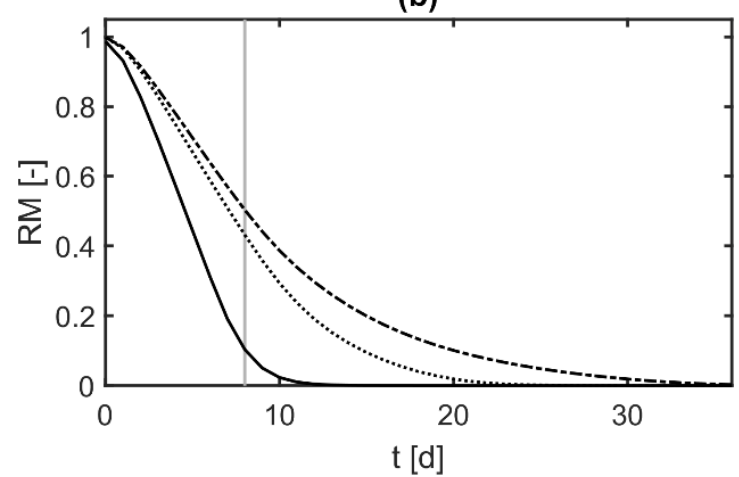

Figure 9. Temporal evolution of the relative area occupied by $B^{-}$in the conservative transport cases (a), and of the relative mass of organic contaminant in the reactive transport simulations (b) performed in the 2-D domain considering different electrode configurations (Conf. 1, Conf. 2 and Conf. 3).

The impact of different electrode configurations is also evaluated considering a delivered reactant, $B^{-}$, and a non-charged contaminant, $\operatorname{Org}$, uniformly present in the 2-D domain with a concentration of $2.5 \mathrm{mM}$. The reaction kinetics follows Eq. (18), with a kinetic rate constant $\lambda=1 \times 10^{-6} \mathrm{~m}^{3} /(\mathrm{mol} \mathrm{s})$. Figure $9 \mathrm{~b}$ shows that the contaminant removal efficiency is strongly dependent on the specific electrode configuration. The expected complete degradation of the organic compounds occurs after 12 days in the ideal and 1-D equivalent Conf. 1, whereas 36 and 23 days are needed in Conf. 2 and Conf. 3, respectively.

The 2-D simulations performed underline the importance of dimensionality and electrode configurations in EKR systems. The design of the electrode layout should minimize zones of low electric potential gradient, where reactant distribution is ineffective and, thus, will result in longer remediation times. 


\section{Conclusions}

In this study we have investigated the effects of Coulombic interactions, reaction kinetics, and dimensionality on conservative and reactive transport of charged species in porous media in the context of electrokinetic remediation. We focused our investigation on the study of electromigration, which is typically the dominant mechanism of electrokinetic transport of charged species. We also limited our analysis to fully saturated porous media and we have demonstrated the important effects of the coupled displacement of ions in these systems. Our results show that the concentration of background electrolytes in the pore water can strongly influence the delivery of species from the electrode reservoir, by either limiting or increasing the concentration of the species delivered in the domain. We have derived a simple analytical expression, valid under certain limiting conditions, to estimate the concentration of a charged amendment that can be delivered by electromigration in a porous medium domain. This study also highlights the effects of compound-specific diffusive properties and dimensionality in EK systems. The importance of such effects for natural advective-dispersive transport in groundwater has been highlighted in a few experimental and modeling studies (e.g, Rolle et al., 2013a; Ye et al., 2015a-c). This model-based analysis demonstrates that such aspects are of great importance also when an electric field is imposed and electromigration becomes the dominant transport process. The outcomes of our simulations show that small differences in diffusion coefficients can radically change the shape of propagating fronts, which can assume rarefaction or shock wave patterns in case the diffusivity of the delivered amendment is higher or lower than the dominant background ion. In order to assess the distribution of delivered charged species in electrokinetic systems it is also important to considering multidimensional setups. The results of the multidimensional simulations performed illustrate that the effectiveness of amendment distribution depends on the electrode layout and polarization and that the dimensionality of the transport problem and the specific electrode configurations 
should be taken into account when upscaling 1-D laboratory setups. Finally, we considered electrokinetic reactive systems and the degradation of a non-charged organic compound. We focused on mixing-controlled reactions and we showed that the charge coupling, resulting in limited or enhanced amendment concentrations in the porous medium, plays a key role for the contaminant removal and on the propagation of EK fronts. Furthermore, analyzing the impact of different reaction kinetics we showed that the different mechanisms of EK-mixing compared to dispersive mixing under natural flow-through conditions can lead to situations in which slower reaction kinetics can enhance contaminant removal.

Overall, although the simulations were conducted for simplified systems selected to illustrate fundamental mechanisms, the findings of this study have important implications that should be considered in practical applications of EKR, especially for the ones involving the delivery of amendments to enhance chemical and/or biological contaminant degradation (i.e., EK-Bio and EK-ISCO). In fact, the effects highlighted in this investigation may help improving the design of EKR technology and optimizing the operations of EKR systems during field scale implementation for in-situ remediation of contaminated groundwater.

We envision that future research should prove and quantify the mechanisms illustrated in this modeling study with dedicated controlled experiments and should address additional aspects, including more complex chemical and reactive systems such as the mobilization of charged inorganic contaminants (e.g., heavy metals and metalloids) that can undergo complex interactions with the solid matrix (Masi et al., 2019; Stolze et al., 2019a,b). Furthermore, the investigation should be extended to multidimensional systems including both physical (e.g., Cirpka et al., 2012, 2015; Jung and Navarre-Sitchler, 2018) and chemical heterogeneity (e.g. Battistel et al., 2019; Fakhreddine et al., 2016; Li et al., 2014). Finally, there is a need to extend the investigation to different conditions, including the effect of electroosmotic flow and exploring the impact of charge interactions on electromigration in unsaturated porous media. 


\section{Acknowledgements}

The authors acknowledge the support of the Capital Region of Denmark (EK-modeling project). M.R. also acknowledges the support of the Independent Research Fund Denmark (grant DFF-7017-00130). 


\section{References}

Acar, Y.B., Alshawabkeh, A.N., 1993. Principles of Electrokinetic Remediation. Environ. Sci. Technol. 27, 2638--2647. https://doi.org/10.1021/es00049a002

Alshawabkeh, A.N., Gale, R.J., Ozsu-Acar, E., Bricka, R.M., 1999. Optimization of 2-D Electrode Configuration for Electrokinetic Remediation. J. Soil Contam. 8, 617-635. https://doi.org/10.1080/10588339991339504

Alt-Epping, P., Tournassat, C., Rasouli, P., Steefel, C.I., Mayer, K.U., Jenni, A., Mäder, U., Sengor, S.S., Fernández, R., 2015. Benchmark reactive transport simulations of a column experiment in compacted bentonite with multispecies diffusion and explicit treatment of electrostatic effects. Comput. Geosci. 19, 535-550. https://doi.org/10.1007/s10596-014-9451-x

Appelo, C.A.J., Wersin, P., 2007. Multicomponent diffusion modeling in clay systems with application to the diffusion of tritium, iodide, and sodium in opalinus clay. Environ. Sci. Technol. 41, 5002-5007. https://doi.org/https://doi.org/10.1021/es0629256

Appelo, C.A.J., 2017. Solute transport solved with the Nernst-Planck equation for concrete pores with "free" water and a double layer. Cem. Concr. Res. 101, 102-113. https://doi.org/10.1016/j.cemconres.2017.08.030

Appelo, C.A.J., Van Loon, L.R., Wersin, P., 2010. Multicomponent diffusion of a suite of tracers (HTO, Cl, Br, I, Na, Sr, Cs) in a single sample of Opalinus Clay. Geochim. Cosmochim. Acta. 74, 1201-1219. https://doi.org/10.1016/j.gca.2009.11.013

Appelo, C.A.J., Vinsot, A., Mettler, S., Wechner, S., 2008. Obtaining the porewater composition of a clay rock by modeling the in- and out-diffusion of anions and cations from an in-situ experiment. J. Contam. Hydrol. 101, 67-76.

https://doi.org/10.1016/j.jconhyd.2008.07.009

Battistel, M., Muniruzzaman, M., Onses, F., Lee, J. and Rolle, M., 2019. Reactive fronts in 
chemically heterogeneous porous media: Experimental and modeling investigation of pyrite oxidation. Appl. Geochem. 11, 77-89.

https://doi.org/10.1016/j.apgeochem.2018.10.026

Boudreau, B.P., Meysman, F.J.R., Middelburg, J.J., 2004. Multicomponent ionic diffusion in porewaters: Coulombic effects revisited. Earth Planet. Sci. Lett. 222, 653-666. https://doi.org/10.1016/j.epsl.2004.02.034

Chowdhury, A.I.A., Gerhard, J.I., Reynolds, D., O’Carroll, D.M., 2017. Low Permeability Zone Remediation via Oxidant Delivered by Electrokinetics and Activated by Electrical Resistance Heating: Proof of Concept. Environ. Sci. Technol. 51, 13295-13303. https://doi.org/10.1021/acs.est.7b02231

Cirpka, O.A., Rolle, M., Chiogna, G., De Barros, F.P. and Nowak, W., 2012. Stochastic evaluation of mixing-controlled steady-state plume lengths in two-dimensional heterogeneous domains. J. Contam. Hydrol. 138, 22-39. https://doi.org/10.1016/j.jconhyd.2012.05.007

Cirpka, O.A., Chiogna, G., Rolle, M., Bellin, A., 2015. Transverse mixing in threedimensional nonstationary anisotropic heterogeneous porous media. Water Resour. Res. 51(1), 241-260. https://doi.org/10.1002/2014WR015331

Fakhreddine, S., Lee, J., Kitanidis, P.K., Fendorf, S., Rolle, M., 2016. Imaging geochemical heterogeneities using inverse reactive transport modeling: An example relevant for characterizing arsenic mobilization and distribution. Adv. Water Resour. 88, 186-197. https://doi.org/10.1016/j.advwatres.2015.12.005

Fan, G., Cang, L., Gomes, H.I., Zhou, D., 2016. Electrokinetic delivery of persulfate to remediate PCBs polluted soils: Effect of different activation methods. Chemosphere 144, 138-147. https://doi.org/10.1016/j.chemosphere.2015.08.074

Gill, R.T., Harbottle, M.J., Smith, J.W.N., Thornton, S.F., 2014. Electrokinetic-enhanced 
bioremediation of organic contaminants: A review of processes and environmental applications. Chemosphere 107, 31-42.

https://doi.org/10.1016/j.chemosphere.2014.03.019

Gill, R.T., Thornton, S.F., Harbottle, M.J., Smith, J.W., 2016. Effect of physical heterogeneity on the electromigration of nitrate in layered granular porous media. Electrochim. Acta. 199, 59-69. https://doi.org/10.1016/j.electacta.2016.02.191

Gill, R.T., Thornton, S.F., Harbottle, M.J., Smith, J.W.N., 2015. Electrokinetic Migration of Nitrate Through Heterogeneous Granular Porous Media. Groundw. Monit. Remediat. 35, 46-56. https://doi.org/10.1111/gwmr.12107

Hochstetler, D.L., Kitanidis, P.K., 2013. The behavior of effective rate constants for bimolecular reactions in an asymptotic transport regime. J. Contam. Hydrol. 144, 88-98. https://doi.org/10.1016/j.jconhyd.2012.10.002

Hochstetler, D.L., Rolle, M., Chiogna, G., Haberer, C.M., Grathwohl, P., Kitanidis, P.K., 2013. Effects of compound-specific transverse mixing on steady-state reactive plumes: Insights from pore-scale simulations and Darcy-scale experiments. Adv. Water Resour. 54, 1-10. https://doi.org/10.1016/j.advwatres.2012.12.007

Hodges, D., Fourie, A., Thomas, D., Reynolds, D., 2013. Overcoming Permanganate Stalling during Electromigration. J. Environ. Eng. 139, 677-684. https://doi.org/10.1061/(ASCE)EE.1943-7870.0000660

Hyldegaard, B.H., Jakobsen, R., Weeth, E.B., Overheu, N.D., Gent, D.B., Ottosen, L.M., 2019. Challenges in electrochemical remediation of chlorinated solvents in natural groundwater aquifer settings. J. Hazard. Mater. 368, 680-688. https://doi.org/10.1016/j.jhazmat.2018.12.064

Jacobs, R.A., Probstein, R.F., 1996. Two-Dimensional Modeling of Electroremediation. AIChE J. 42, 1685-1696. https://doi.org/10.1002/aic.690420620 
Jung, H.B., Yang, J.S., Um, W., 2015. Bench-scale electrokinetic remediation for cesiumcontaminated sediment at the Hanford Site, USA. J. Radioanal. Nucl. Chem. 304, 615625. https://doi.org/10.1007/s10967-014-3852-0

Jung, H. and Navarre-Sitchler, A., 2018. Scale effect on the time-dependence of mineral dissolution rates in physically heterogeneous porous media. Geochimica et Cosmochimica Acta. 231, 70-83. https://doi.org/10.1016/j.gca.2018.05.009

Kim, D.-H., Ryu, B.-G., Park, S.-W., Seo, C.-I., Baek, K., 2009. Electrokinetic remediation of $\mathrm{Zn}$ and Ni-contaminated soil. J. Hazard. Mater. 165, 501-505. https://doi.org/10.1016/j.jhazmat.2008.10.025

Kim, W.-S., Park, G.-Y., Kim, D.-H., Jung, H.-B., Ko, S.-H., Baek, K., 2012. In situ field scale electrokinetic remediation of multi-metals contaminated paddy soil: Influence of electrode configuration. Electrochim. Acta 86, 89-95. https://doi.org/10.1016/j.electacta.2012.02.078

Kim, W.S., Nam, S., Chang, S., Kim, H.J., Um, W., 2018. Removal of Chalk River unidentified deposit (CRUD) radioactive waste by enhanced electrokinetic process. J. Ind. Eng. Chem. 57, 89-96. https://doi.org/10.1016/j.jiec.2017.08.011

Krabbenhøft, K., Krabbenhøft, J., 2008. Application of the Poisson-Nernst-Planck equations to the migration test. Cem. Concr. Res. 38, 77-88. https://doi.org/10.1016/j.cemconres.2007.08.006

Li, L., Salehikhoo, F., Brantley, S.L., Heidari, P., 2014. Spatial zonation limits magnesite dissolution in porous media. Geochim. Cosmochim. Acta. 126, 555-573. https://doi.org/10.1016/j.gca.2013.10.051

Li, Z., Yu, J.W., Neretnieks, I., 1996. A new approach to electrokinetic remediation of soils polluted by heavy metals. J. Contam. Hydrol. 22, 241-253. https://doi.org/10.1016/0169-7722(95)00093-3 
Lima, A.T., Hofmann, A., Reynolds, D., Ptacek, C.J., Van Cappellen, P., Ottosen, L.M., Pamukcu, S., Alshawabekh, A., O’Carroll, D.M., Riis, C., Cox, E., Gent, D.B., Landis, R., Wang, J., Chowdhury, A.I.A., Secord, E.L., Sanchez-Hachair, A., 2017. Environmental Electrokinetics for a sustainable subsurface. Chemosphere. 181, 122133. https://doi.org/10.1016/j.chemosphere.2017.03.143

Liu, C., Shang, J., Zachara, J.M., 2011. Multispecies diffusion models: A study of uranyl species diffusion. Water Resour. Res. 47(12). https://doi.org/10.1029/2011WR010575

Lohner, S.T., Katzoreck, D., Tiehm, A., 2008a. Electromigration of microbial electron acceptors and nutrients: (I) Transport in synthetic media. J. Environ. Sci. Heal. Part A 43, 913-921. https://doi.org/10.1080/10934520801974434

Lohner, S.T., Katzoreck, D., Tiehm, A., 2008b. Electromigration of microbial electron acceptors and nutrients: (II) Transport in groundwater. J. Environ. Sci. Heal. Part A 43, 922-925. https://doi.org/10.1080/10934520801974442

López-Vizcaíno, R., Risco, C., Isidro, J., Rodrigo, S., Saez, C., Cañizares, P., Navarro, V., Rodrigo, M.A., 2017a. Scale-up of the electrokinetic fence technology for the removal of pesticides. Part I: Some notes about the transport of inorganic species. Chemosphere. 166, 540-548. https://doi.org/10.1016/j.chemosphere.2016.09.113

López-Vizcaíno, R., Risco, C., Isidro, J., Rodrigo, S., Saez, C., Cañizares, P., Navarro, V., Rodrigo, M.A., 2017b. Scale-up of the electrokinetic fence technology for the removal of pesticides. Part II: Does size matter for removal of herbicides? Chemosphere. 166, 549-555. https://doi.org/10.1016/j.chemosphere.2016.09.114

López-Vizcaíno, R., Yustres, A., León, M.J., Saez, C., Cañizares, P., Rodrigo, M.A., Navarro, V., 2017c. Multiphysics Implementation of Electrokinetic Remediation Models for Natural Soils and Porewaters. Electrochim. Acta 225, 93-104. https://doi.org/10.1016/j.electacta.2016.12.102 
MacGillivray, A.D., 1968. Nernst-Planck Equations and the Electroneutrality and Donnan Equilibrium Assumptions. J. Chem. Phys. 48, 2903-2907. https://doi.org/10.1063/1.1669549

Mao, X., Shao, X., Zhang, Z., 2019. Pilot-scale electro-kinetic remediation of lead polluted field sediments: model designation, numerical simulation, and feasibility evaluation. Environ. Sci. Eur. 31(1), 25. https://doi.org/10.1186/s12302-019-0209-x

Mao, X., Wang, J., Ciblak, A., Cox, E.E., Riis, C., Terkelsen, M., Gent, D.B., Alshawabkeh, A.N., 2012. Electrokinetic-enhanced bioaugmentation for remediation of chlorinated solvents contaminated clay. J. Hazard. Mater. 213, 311-317. https://doi.org/10.1016/j.jhazmat.2012.02.001

Masi, M., Ceccarini, A., Iannelli, R., 2017a. Model-based optimization of field-scale electrokinetic treatment of dredged sediments. Chem. Eng. J. 328, 87-97. https://doi.org/10.1016/J.CEJ.2017.07.004

Masi, M., Ceccarini, A., Iannelli, R., 2017b. Multispecies reactive transport modelling of electrokinetic remediation of harbour sediments. J. Hazard. Mater. 326, 187-196. https://doi.org/10.1016/j.jhazmat.2016.12.032

Masi, M., Paz-Garcia, J.M., Gomez-Lahoz, C., Villen-Guzman, M., Ceccarini, A., Iannelli, R., 2019. Modeling of electrokinetic remediation combining local chemical equilibrium and chemical reaction kinetics. J. Hazard. Mater. 371, 728-733. https://doi.org/10.1016/j.jhazmat.2019.03.014

Mattson, E.D., Bowman, R.S., Lindgren, E.R., 2002a. Electrokinetic ion transport through unsaturated soil: 1. Theory, model development, and testing. J. Contam. Hydrol. 54, 99120. https://doi.org/10.1016/S0169-7722(01)00144-9

Mattson, E.D., Bowman, R.S., Lindgren, E.R., 2002b. Electrokinetic ion transport through unsaturated soil: 2. Application to a heterogeneous field site. J. Contam. Hydrol. 54, 
121-140. https://doi.org/10.1016/S0169-7722(01)00145-0

Muniruzzaman, M., Haberer, C.M., Grathwohl, P., Rolle, M., 2014. Multicomponent ionic dispersion during transport of electrolytes in heterogeneous porous media: Experiments and model-based interpretation. Geochim. Cosmochim. Acta 141, 656--669. https://doi.org/10.1016/j.gca.2014.06.020

Muniruzzaman, M., Rolle, M., 2017. Experimental investigation of the impact of compoundspecific dispersion and electrostatic interactions on transient transport and solute breakthrough. Water Resour. Res. 53, 1189-1209. https://doi.org/10.1002/2016WR019727

Muniruzzaman, M., Rolle, M., 2016. Modeling multicomponent ionic transport in groundwater with IPhreeqc coupling: Electrostatic interactions and geochemical reactions in homogeneous and heterogeneous domains. Adv. Water Resour. 98, 1-15. https://doi.org/10.1016/j.advwatres.2016.10.013

Muniruzzaman, M., Rolle, M., 2015. Impact of multicomponent ionic transport on pH fronts propagation in saturated porous media. Water Resour. Res. 51, 6739-6755. https://doi.org/10.1002/2015WR017134

Newman, J., Thomas-Alyea, K.E., 2004. Electrochemical systems. John Wiley \& Sons

Ottosen, L.M., Larsen, T.H., Jensen, P.E., Kirkelund, G.M., Kerrn-Jespersen, H., Tuxen, N., Hyldegaard, B.H., 2019. Electrokinetics applied in remediation of subsurface soil contaminated with chlorinated ethenes - A review. Chemosphere. 235, 113-125. https://doi.org/10.1016/j.chemosphere.2019.06.075

Pamukcu, S., 2009. Electrochemical Transport and Transformations, in: Electrochemical Remediation Technologies for Polluted Soils, Sediments and Groundwater. John Wiley \& Sons, Inc., Hoboken, NJ, USA, pp. 29-64. https://doi.org/10.1002/9780470523650.ch2 
Parker, B.L., Chapman, S.W., Guilbeault, M.A., 2008. Plume persistence caused by back diffusion from thin clay layers in a sand aquifer following TCE source-zone hydraulic isolation. J. Contam. Hydrol. 102, 86-104. https://doi.org/10.1016/j.jconhyd.2008.07.003

Parkhurst, D.L. and Wissmeier, L., 2015. PhreeqcRM: A reaction module for transport simulators based on the geochemical model PHREEQC, Adv. Water Resour. 83, 176189. https://doi.org/10.1016/j.advwatres.2015.06.001

Paz-García, J.M., Johannesson, B., Ottosen, L.M., Ribeiro, A.B., Rodríguez-Maroto, J.M., 2011. Modeling of electrokinetic processes by finite element integration of the NernstPlanck-Poisson system of equations. Sep. Purif. Technol. 79, 183-192. https://doi.org/10.1016/j.seppur.2011.02.023

Paz-García, J.M., Villén-Guzmán, M., García-Rubio, A., Hall, S., Ristinmaa, M., GómezLahoz, C., 2016. A Coupled Reactive-Transport Model for Electrokinetic Remediation, in: Electrokinetics Across Disciplines and Continents. Springer International Publishing, Cham. 251-278. https://doi.org/10.1007/978-3-319-20179-5_13

Pazos, M., Gouveia, S., Sanromán, M.A., Cameselle, C., 2008. Electromigration of Mn, Fe, $\mathrm{Cu}$ and $\mathrm{Zn}$ with citric acid in contaminated clay. J. Environ. Sci. Heal. - Part A Toxic/Hazardous Subst. Environ. Eng. 43, 823-831. https://doi.org/10.1080/10934520801974004

Rasa, E., Chapman, S.W., Bekins, B.A., Fogg, G.E., Scow, K.M., MacKay, D.M., 2011. Role of back diffusion and biodegradation reactions in sustaining an MTBE/TBA plume in alluvial media. J. Contam. Hydrol. 126, 235-247. https://doi.org/10.1016/j.jconhyd.2011.08.006

Rasouli, P., 2016. On the role of multicomponent diffusion and electrochemical migration for reactive transport in porous media. University of British Columbia. 
https://doi.org/10.14288/1.0340304

Rasouli, P., Steefel, C.I., Mayer, K.U., Rolle, M., 2015. Benchmarks for multicomponent diffusion and electrochemical migration. Comput. Geosci. 19, 523-533. https://doi.org/10.1007/s10596-015-9481-z

Reddy, K.R., Cameselle, C., 2009. Overview of Electrochemical Remediation Technologies, in: Electrochemical Remediation Technologies for Polluted Soils, Sediments and Groundwater. John Wiley \& Sons, Inc., Hoboken, NJ, USA, pp. 1-28. https://doi.org/10.1002/9780470523650.ch1

Reddy, K.R., Chaparro, C., Saichek, R.E., 2003. Removal of mercury from clayey soils using electrokinetics. J. Environ. Sci. Heal. - Part A Toxic/Hazardous Subst. Environ. Eng. 38, 307-338. https://doi.org/10.1081/ESE-120016897

Reynolds, D.A., Jones, E.H., Gillen, M., Yusoff, I., Thomas, D.G., 2008. Electrokinetic migration of permanganate through low-permeability media. Ground Water 46, 629637. https://doi.org/10.1111/j.1745-6584.2008.00415.x

Ribeiro, A.B., Mexia, J.T., 1997. A dynamic model for the electrokinetic removal of copper from a polluted soil. J. Hazard. Mater. 56, 257-271. https://doi.org/10.1016/S03043894(97)00060-5

Risco, C., López-Vizcaíno, R., Sáez, C., Yustres, A., Cañizares, P., Navarro, V., Rodrigo, M.A., 2016. Remediation of soils polluted with 2,4-D by electrokinetic soil flushing with facing rows of electrodes: A case study in a pilot plant. Chem. Eng. J. 285, 128 136. https://doi.org/10.1016/j.cej.2015.10.011

Rolle, M., Chiogna, G., Hochstetler, D.L., Kitanidis, P.K., 2013a. On the importance of diffusion and compound-specific mixing for groundwater transport: An investigation from pore to field scale. J. Contam. Hydrol. 153, 51-68. https://doi.org/10.1016/j.jconhyd.2013.07.006 
Rolle, M., Kitanidis, P.K., 2014. Effects of compound-specific dilution on transient transport and solute breakthrough: A pore-scale analysis. Adv. Water Resour. 71, 186-199. https://doi.org/10.1016/j.advwatres.2014.06.012

Rolle, M., Muniruzzaman, M., Haberer, C.M., Grathwohl, P., 2013b. Coulombic effects in advection-dominated transport of electrolytes in porous media: Multicomponent ionic dispersion. Geochim. Cosmochim. Acta 120, 195--205. https://doi.org/10.1016/j.gca.2013.06.031

Rolle, M., Sprocati, R., Masi, M., Jin, B., Muniruzzaman, M., 2018. Nernst-Planck-based Description of Transport, Coulombic Interactions, and Geochemical Reactions in Porous Media: Modeling Approach and Benchmark Experiments. Water Resour. Res. 54, 31763195. https://doi.org/10.1002/2017WR022344

Sastre, M., Santaballa, J.A., 1989. A note on the meaning of the electroneutrality condition for solutions. J. Chem. Educ. 66, 403. https://doi.org/10.1021/ed066p403

Schultz, D.S., 1997. Electroosmosis technology for soil remediation: Laboratory results, field trial, and economic modeling. J. Hazard. Mater. 55, 81-91. https://doi.org/10.1016/S0304-3894(97)00014-9

Sprocati, R., Masi, M., Muniruzzaman, M., Rolle, M., 2019. Modeling electrokinetic transport and biogeochemical reactions in porous media: a multidimensional NernstPlanck-Poisson approach with PHREEQC coupling. Adv. Water Resour. 127, 134-147. https://doi.org/10.1016/j.advwatres.2019.03.011

Stolze, L., Zhang, D., Guo, H. \& Rolle, M., 2019a. Surface complexation modeling of arsenic mobilization from goethite: Interpretation of an in-situ experiment. Geochim. Cosmochim. Acta. 248, 274-288. https://doi.org/10.1016/j.gca.2019.01.008

Stolze, L., Zhang, D., Guo, H. \& Rolle, M., 2019b. Model-based interpretation of groundwater arsenic mobility during in situ reductive transformation of ferrihydrite. 
Environ. Sci. Technol. 53, 6845-6854. https://doi.org/10.1021/acs.est.9b00527

Thevanayagam, S., Rishindran, T., 1998. Injection of Nutrients and TEAs in Clayey Soils

Using Electrokinetics. J. Geotech. Geoenvironmental Eng. 124, 330-338.

https://doi.org/10.1061/(asce)1090-0241(1998)124:4(330)

Virkutyte, J., Sillanpää, M., Latostenmaa, P., 2002. Electrokinetic soil remediation - Critical overview. Sci. Total Environ. 289, 97-121. https://doi.org/10.1016/S00489697(01)01027-0

Wu, M.Z., Reynolds, D.A., Fourie, A., Prommer, H., Thomas, D.G., 2012a. Electrokinetic in situ oxidation remediation: Assessment of parameter sensitivities and the influence of aquifer heterogeneity on remediation efficiency. J. Contam. Hydrol. 136-137, 72-85. https://doi.org/10.1016/j.jconhyd.2012.04.005

Wu, M.Z., Reynolds, D.A., Fourie, A., Thomas, D.G., 2013. Optimal Field Approaches for Electrokinetic In Situ Oxidation Remediation. Groundw. Monit. Remediat. 33, 62-74. https://doi.org/10.1111/j.1745-6592.2012.01410.x

Wu, M.Z., Reynolds, D.A., Prommer, H., Fourie, A., Thomas, D.G., 2012b. Numerical evaluation of voltage gradient constraints on electrokinetic injection of amendments. Adv. Water Resour. 38, 60-69. https://doi.org/10.1016/j.advwatres.2011.11.004

Yang, M., Annable, M.D., Jawitz, J.W., 2017. Field-scale forward and back diffusion through low-permeability zones. J. Contam. Hydrol. 202, 47-58. https://doi.org/10.1016/j.jconhyd.2017.05.001

Ye, Y., Chiogna, G., Cirpka, O.A., Grathwohl, P., Rolle, M., 2015a. Experimental investigation of compound-specific dilution of solute plumes in saturated porous media: 2-D vs. 3-D flow-through systems. J. Contam. Hydrol. 172, 33-47. https://doi.org/10.1016/j.jconhyd.2014.11.002

Ye, Y., Chiogna, G., Cirpka, O.A., Grathwohl, P., Rolle, M., 2015b. Enhancement of plume 
dilution in two-dimensional and three-dimensional porous media by flow focusing in high-permeability inclusions. Water Resour. Res. 51, 5582-5602. https://doi.org/10.1002/2015WR016962

Ye, Y., Chiogna, G., Cirpka, O.A., Grathwohl, P., Rolle, M., 2015c, Experimental evidence of helical flow in porous media, Phys. Rev. Lett., 115(19), 194502. https://doi.org/10.1103/PhysRevLett.115.194502

Yustres, López-Vizcaíno, R., Sáez, C., Cañizares, P., Rodrigo, M.A., Navarro, V., 2018. Water transport in electrokinetic remediation of unsaturated kaolinite. Experimental and numerical study. Sep. Purif. Technol. 192, 196-204. https://doi.org/10.1016/j.seppur.2017.10.009

Zhongming, L., Yu, J.W., Neretnieks, I., 1997. Removal of Pb(II), Cd(II) and Cr(III) from sand by electromigration. J. Hazard. Mater. 55, 295-304. https://doi.org/10.1016/S03043894(97)00021-6 